\title{
Robust a posteriori error estimation for mixed finite element approximation of linear poroelasticity
}

DOI:

10.1093/imanum/draa058

\section{Document Version}

Accepted author manuscript

Link to publication record in Manchester Research Explorer

\section{Citation for published version (APA):}

Khan, A., \& Silvester, D. (2020). Robust a posteriori error estimation for mixed finite element approximation of linear poroelasticity. IMA Journal of Numerical Analysis. https://doi.org/10.1093/imanum/draa058

\section{Published in:}

IMA Journal of Numerical Analysis

\section{Citing this paper}

Please note that where the full-text provided on Manchester Research Explorer is the Author Accepted Manuscript or Proof version this may differ from the final Published version. If citing, it is advised that you check and use the publisher's definitive version.

\section{General rights}

Copyright and moral rights for the publications made accessible in the Research Explorer are retained by the authors and/or other copyright owners and it is a condition of accessing publications that users recognise and abide by the legal requirements associated with these rights.

\section{Takedown policy}

If you believe that this document breaches copyright please refer to the University of Manchester's Takedown Procedures [http://man.ac.uk/04Y6Bo] or contact uml.scholarlycommunications@manchester.ac.uk providing relevant details, so we can investigate your claim.

\section{OPEN ACCESS}


IMA Journal of Numerical Analysis (2020) Page 1 of 25 doi:10.1093/imanum/drnxxx

\title{
Robust a posteriori error estimation for mixed finite element approximation of linear poroelasticity
}

\author{
ARBAZ KHAN† \\ Department of Mathematics, Indian Institute of Technology Roorkee (IITR), \\ Roorkee, India \\ AND \\ DAVID J. SILVESTER $\ddagger$ \\ Department of Mathematics, University of Manchester, M13 9PL, UK
}

[Received on 20 July 2020]

This work is dedicated to the memory of John W. Barrett, who introduced the concept of inf-sup stability to the corresponding author in the bar at the MAFELAP conference in 1981.

We analyze a posteriori error estimators for locking-free mixed finite element approximation of Biot's consolidation model. Three estimators are described. The simplest of these is a conventional residualbased estimator. We establish bounds relating the estimated and true errors and show that these are independent of the physical parameters. The other two estimators require the solution of local problems. These local problem estimators are also shown to be reliable, efficient and robust. Numerical results are presented that validate the theoretical estimates and illustrate the effectiveness of the estimators in guiding adaptive solution algorithms. The IFISS and T-IFISS software packages used for the computational experiments are available online.

Keywords: Biot's consolidation model, mixed approximation, locking-free, a posteriori analysis, error estimation, adaptivity.

\section{Introduction}

Mathematical models of fluid flow and deformation in porous media have wide-ranging applications in science and engineering. The focus here is on Biot's consolidation model. This is the starting point for modelling human organs in computational medicine and for modelling the mechanics of permeable rock in geophysics. Finite element methods for Biot's consolidation model have been widely studied over the last four decades. We refer to Reed (1984); Vermeer \& Verruijt (1981); Zienkiewicz \& Shiomi (1984) for the various primal methods, Murad \& Loula (1992, 1994); Murad et al. (1996) for mixed methods, Korsawe \& Starke (2005) for Galerkin least squares methods, Chen et al. (2013) for discontinuous Galerkin methods and Phillips \& Wheeler (2007a,b, 2008); Yi (2013) for combination methods. The key requirement for any numerical approximation strategy is to ensure inf-sup stability, so that spurious pressure modes associated with volumetric locking are avoided.

The general locking-free formulation of Biot's consolidation model analysed in Oyarzúa \& RuizBaier (2016) is the starting point for our study. Following their approach, inf-sup stability is assured

\footnotetext{
†Email: arbaz@ma.iitr.ac.in

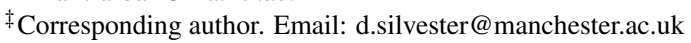


by including an additional scalar unknown into the classical Biot model (the so-called total pressure), representing the volumetric part of the total stress. A slightly simplified version is the following threefield model: for a given body force $f$ and a volumetric source (or sink) $g$, we seek the displacement of the elastic medium $\boldsymbol{u}$, the pore pressure of the fluid $p_{F}$ and the total pressure $p_{T}=-\lambda \nabla \cdot \boldsymbol{u}+\alpha p_{F}$ satisfying

$$
\begin{aligned}
-\nabla \cdot \sigma & =\boldsymbol{f} & & \text { in } \Omega, \\
-\nabla \cdot \boldsymbol{u}-\frac{1}{\lambda}\left(p_{T}-\alpha p_{F}\right) & =0 & & \text { in } \Omega, \\
\frac{1}{\lambda}\left(\alpha p_{T}-2 \alpha^{2} p_{F}\right)+\nabla \cdot\left(\kappa \nabla p_{F}\right) & =g & & \text { in } \Omega, \\
\sigma \boldsymbol{n} & =\mathbf{0} & & \text { on } \Gamma_{p}, \\
p_{F} & =h & & \text { on } \Gamma_{p}, \\
\boldsymbol{u} & =\boldsymbol{g} & & \text { on } \Gamma_{\boldsymbol{u}}, \\
\left(\kappa \nabla p_{F}\right) \cdot \boldsymbol{n} & =0 & & \text { on } \Gamma_{\boldsymbol{u}} .
\end{aligned}
$$

We will assume that the region of interest $\Omega$, is a bounded Lipschitz polygon in $\mathbb{R}^{2}$ (polyhedral in $\mathbb{R}^{3}$ ) with a boundary $\Gamma=\partial \Omega=\bar{\Gamma}_{\boldsymbol{u}} \cup \bar{\Gamma}_{p}$ and $\Gamma_{\boldsymbol{u}} \cap \Gamma_{p}=\emptyset$. In this formulation, (1.1a) represents the force equilibrium (conservation of momentum), (1.1b) represents the pressure balance and (1.1c) represents conservation of mass. As usual, $\boldsymbol{\sigma}=2 \mu \boldsymbol{\varepsilon}(\boldsymbol{u})-p_{T} \mathrm{I}$, (where I is the $2 \times 2$ matrix) represents the stress tensor and $\boldsymbol{\varepsilon}(\boldsymbol{u})=\left(\nabla \boldsymbol{u}+(\nabla \boldsymbol{u})^{\top}\right) / 2$ is the strain tensor. We also note that the system (1.1) represents a static problem associated with a single step of an implicit time integrator applied to the underlying evolution equations.

The model (1.1) is a simplified version of the standard Biot model in that the constrained specific storage coefficient (denoted by $c_{0}$ in Oyarzúa \& Ruiz-Baier (2016)) has been replaced by the ratio $\alpha^{2} / \lambda$. This replacement is justified by Lee et al. (2017) by consideration of practical situations where the compressibility of the fluid is close to zero and where $\mu \lesssim \lambda$. In this simplified model, $\kappa=k_{t} \kappa_{1}$ is a scaled hydraulic conductivity, where $k_{t}>0$ is the time step; $0<\alpha \leqslant 1$ is a parameter representing a scaled Biot-Willis constant and $\lambda, \mu$ represent scaled Lamé parameters with $\mu \sim 1$ and $1 \leqslant \lambda<\infty$. Volumetric locking is associated with practical situations where $\lambda \gg 1$. The stability of the formulation (1.1) is discussed at length in Lee et al. (2017) along with robust preconditioning strategies for solving the associated spatially-discretised matrix systems.

The focus in this work is on local (a posteriori) error estimation and adaptive solution algorithms. Our contribution extends the pioneering work of Ern \& Meunier (2009). It also complements the recent studies of Ahmed et al. (2019, 2020), Li \& Zikatanov (2019) and Oyarzúa et al. (2019). Three different types of error estimation strategies are considered herein. The first is the classical residual-based a posteriori error estimator and the other two are based on solving local problems. Upper and lower bounds are established ensuring the robustness and efficiency of the three estimation strategies. The key point is that the constants in these bounds are independent of the local mesh parameters $\left(h_{E}\right.$ and $\left.h_{K}\right)$, the Lamé parameters $(\mu$ and $\lambda$ ), the Biot-Willis parameter $(\alpha)$ and the scaled hydraulic conductivity parameter $(\kappa)$.

The paper is organised as follows: Section 2 reviews the well posedness of the weak formulation corresponding to (1.1) and discusses viable approximation spaces for the component fields. The theoretical basis for the local error estimation strategies is reviewed in Section 3. The most important contribution is a rigorous verification that the approximation error can be reliably and efficiently estimated by 
solving three uncoupled scalar elliptic (local) problems. Computational results presented in Section 4 validate the theoretical estimates and illustrate the effectiveness of the estimation strategies in guiding an efficient adaptive refinement algorithm. In the sequel we use the symbols $\lesssim$ and $\gtrsim$ to denote bounds which are valid up to positive constants that are independent of the parameters $h_{E}, h_{K}, \mu, \lambda, \alpha$ and $\kappa$.

\section{Mixed approximation}

Let $H^{s}(\omega)$ denote the usual Sobolev spaces with the associated norm $\|\cdot\|_{s, \omega}$ for $s \geqslant 0$. In the case $\omega=\Omega$, we use $H^{s}$ instead of $H^{s}(\Omega)$ and $\|\cdot\|_{s}$ instead of $\|\cdot\|_{s, \Omega}$. We will denote vector-valued Sobolev spaces by boldface letters $\boldsymbol{H}^{s}(\boldsymbol{\omega})=\boldsymbol{H}^{s}\left(\boldsymbol{\omega} ; \mathbb{R}^{2}\right)$. We let $(\cdot, \cdot)$ denote the standard $L^{2}$-inner product, and we define solution spaces

$$
\begin{aligned}
& \boldsymbol{H}_{\Gamma_{\boldsymbol{u}}}^{1}:=\left\{\boldsymbol{v} \in \boldsymbol{H}^{1}(\Omega)|\boldsymbol{v}|_{\Gamma_{\boldsymbol{u}}}=\boldsymbol{g}\right\}, \boldsymbol{H}^{\frac{1}{2}}\left(\Gamma_{\boldsymbol{u}}\right):=\left\{\boldsymbol{v}|\boldsymbol{v}=\boldsymbol{u}|_{\Gamma_{\boldsymbol{u}}}, \boldsymbol{u} \in \boldsymbol{H}^{1}(\Omega)\right\}, \\
& H_{\Gamma_{p}}^{1}:=\left\{q \in H^{1}(\Omega)|q|_{\Gamma_{p}}=h\right\}, \quad H^{\frac{1}{2}}\left(\Gamma_{p}\right):=\left\{q|q=p|_{\Gamma_{p}}, p \in H^{1}(\Omega)\right\},
\end{aligned}
$$

and associated test spaces

$$
\begin{gathered}
\boldsymbol{H}_{\Gamma_{\boldsymbol{u}, 0}}^{1}(\Omega):=\left\{\boldsymbol{v} \in \boldsymbol{H}^{1}(\Omega)|\boldsymbol{v}|_{\Gamma_{\boldsymbol{u}}}=\mathbf{0}\right\}, \quad M:=L^{2}(\Omega), \\
H_{\Gamma_{p, 0}}^{1}(\Omega):=\left\{q \in H^{1}(\Omega)|q|_{\Gamma_{p}}=0\right\} .
\end{gathered}
$$

The standard weak formulation of (1.1) is given by: find $\left(\boldsymbol{u}, p_{T}, p_{F}\right) \in \boldsymbol{H}_{\Gamma_{\boldsymbol{u}}}^{1} \times M \times H_{\Gamma_{p}}^{1}$ such that

$$
\begin{aligned}
a(\boldsymbol{u}, \boldsymbol{v})+b_{1}\left(\boldsymbol{v}, p_{T}\right) & =f(\boldsymbol{v}) & & \forall \boldsymbol{v} \in \boldsymbol{H}_{\Gamma_{\boldsymbol{u}, 0}}^{1}, \\
b_{1}\left(\boldsymbol{u}, q_{T}\right)-c\left(p_{T}, q_{T}\right)+b_{2}\left(p_{F}, q_{T}\right) & =0 & & \forall q_{T} \in M, \\
b_{2}\left(q_{F}, p_{T}\right)-d\left(p_{F}, q_{F}\right) & =g\left(q_{F}\right) & & \forall q_{F} \in H_{\Gamma_{p, 0}}^{1},
\end{aligned}
$$

with forms defined so that

$$
\begin{gathered}
a(\boldsymbol{u}, \boldsymbol{v})=2 \mu \int_{\Omega} \boldsymbol{\varepsilon}(\boldsymbol{u}): \boldsymbol{\varepsilon}(\boldsymbol{v}), \quad b_{1}\left(\boldsymbol{v}, p_{T}\right)=-\int_{\Omega} p_{T} \nabla \cdot \boldsymbol{v}, \quad f(\boldsymbol{v})=\int_{\Omega} \boldsymbol{f} \boldsymbol{v}, \\
c\left(p_{T}, q_{T}\right)=\frac{1}{\lambda} \int_{\Omega} p_{T} q_{T}, \quad b_{2}\left(p_{F}, q_{T}\right)=\frac{\alpha}{\lambda} \int_{\Omega} p_{F} q_{T}, \\
d\left(p_{F}, q_{F}\right)=\frac{2 \alpha}{\lambda} \int_{\Omega} p_{F} q_{F}+\int_{\Omega} \kappa \nabla p_{F} \cdot \nabla q_{F}, \quad g\left(q_{F}\right)=\int_{\Omega} g q_{F} .
\end{gathered}
$$

We will assume that the load function $\boldsymbol{f} \in\left(L^{2}(\Omega)\right)^{2}$. For convenience, the boundary data $\boldsymbol{g} \in \boldsymbol{H}^{\frac{1}{2}}\left(\Gamma_{\boldsymbol{u}}\right)$ and $h \in H^{\frac{1}{2}}\left(\Gamma_{p}\right)$ will be assumed to be a polynomial of sufficiently high degree in each component-this will ensure that no error is incurred in approximating the essential boundary condition on $\Gamma_{u}$ and $\Gamma_{p}$. We also assume that $\kappa>0$ is a given, scalar, piecewise constant function on $\Omega$. Specifically, $\kappa$ is equal to the constant $\kappa_{K}$ on each $K \in \mathscr{T}_{h}$, where $\left\{\mathscr{T}_{h}\right\}$ denotes a family of shape regular rectangular/triangular meshes of $\bar{\Omega}$ into rectangles/triangles $K$ of diameter $h_{K}$. Moreover, following convention, we define the bilinear form

$$
\begin{array}{r}
\mathscr{B}\left(\boldsymbol{u}, p_{T}, p_{F} ; \boldsymbol{v}, q_{T}, q_{F}\right)=a(\boldsymbol{u}, \boldsymbol{v})+b_{1}\left(\boldsymbol{v}, p_{T}\right)+b_{1}\left(\boldsymbol{u}, q_{T}\right)-c\left(p_{T}, q_{T}\right) \\
+b_{2}\left(p_{F}, q_{T}\right)+b_{2}\left(p_{T}, q_{F}\right)-d\left(p_{F}, q_{F}\right),
\end{array}
$$


so as to express the formulation (2.1) in a compact form: find $\left(\boldsymbol{u}, p_{T}, p_{F}\right) \in \boldsymbol{H}_{\Gamma_{\boldsymbol{u}}}^{1} \times M \times H_{\Gamma_{p}}^{1}$ such that

$$
\mathscr{B}\left(\boldsymbol{u}, p_{T}, p_{F} ; \boldsymbol{v}, q_{T}, q_{F}\right)=f(\boldsymbol{v})+g\left(q_{F}\right), \quad \forall\left(\boldsymbol{v}, q_{T}, q_{F}\right) \in \boldsymbol{H}_{\Gamma_{\boldsymbol{u}, 0}}^{1} \times M \times H_{\Gamma_{p, 0}}^{1} .
$$

The error analysis will be developed in the parameter-dependent (energy) norm

$$
\left\|\left(\boldsymbol{u}, p_{T}, p_{F}\right)\right\|^{2}=2 \mu\|\nabla \boldsymbol{u}\|_{0}^{2}+\left(\frac{1}{2 \mu}+\frac{1}{\lambda}\right)\left\|p_{T}\right\|_{0}^{2}+\frac{\alpha^{2}}{\lambda}\left\|p_{F}\right\|_{0}^{2}+\left\|\kappa^{1 / 2} \nabla p_{F}\right\|_{0}^{2} .
$$

We will use the following estimates (Ainsworth \& Oden, 2000; Boffi et al., 2013):

$$
\begin{gathered}
a(\boldsymbol{v}, \boldsymbol{v}) \geqslant C_{K} 2 \mu\|\nabla \boldsymbol{v}\|_{0}^{2} \quad \forall \boldsymbol{v} \in \boldsymbol{H}_{\Gamma_{\boldsymbol{u}, 0}}^{1}, \\
\inf _{0 \neq q \in M} \sup _{0 \neq \boldsymbol{v} \in \boldsymbol{H}_{\Gamma_{\boldsymbol{u}, 0}^{1}}} \frac{\left|b_{1}(\boldsymbol{v}, q)\right|}{\|\nabla \boldsymbol{v}\|_{0}\|q\|_{0}} \geqslant C_{\Omega}, \\
a(\boldsymbol{u}, \boldsymbol{v}) \leqslant 2 \mu\|\nabla \boldsymbol{u}\|_{0}\|\nabla \boldsymbol{v}\|_{0} \quad \forall \boldsymbol{u}, \boldsymbol{v} \in \boldsymbol{H}_{\Gamma_{\boldsymbol{u}, 0}}^{1} .
\end{gathered}
$$

The following theorem establishes the stability of the three-field formulation (2.1).

THEOREM 2.1 For any $\left(\boldsymbol{u}, p_{T}, p_{F}\right) \in \boldsymbol{H}_{\Gamma \boldsymbol{u}, 0}^{1} \times M \times H_{\Gamma_{p, 0}}^{1}$, there exists a triplet of functions $\left(\boldsymbol{v}, q_{T}, q_{F}\right) \in$ $\boldsymbol{H}_{\Gamma_{\boldsymbol{u}, 0}}^{1} \times M \times H_{\Gamma_{p, 0}}^{1}$, with $\left\|\left|\left(\boldsymbol{v}, q_{T}, q_{F}\right)\left\|\left|\lesssim\left\|\left|\left(\boldsymbol{u}, p_{T}, p_{F}\right)\|\mid\|\right.\right.\right.\right.\right.\right.$, satisfying

$$
\mathscr{B}\left(\boldsymbol{u}, p_{T}, p_{F} ; \boldsymbol{v}, q_{T}, q_{F}\right) \gtrsim\left\|\left|\left\|\left(\boldsymbol{u}, p_{T}, p_{F}\right) \mid\right\|^{2} .\right.\right.
$$

Proof. The structure of the proof is standard. We include it for completeness and also to keep track of the constants. First, since $p_{T} \in M=L^{2}(\Omega)$, a consequence of the condition (2.6) is that there exists a function $\boldsymbol{v} \in \boldsymbol{H}_{\Gamma_{\boldsymbol{u}, 0}}^{1}$ satisfying

$$
\left(p_{T}, \nabla \cdot v\right) \geqslant C_{\Omega}(2 \mu)^{-1}\left\|p_{T}\right\|_{0}^{2}, \quad(2 \mu)^{1 / 2}\|\nabla v\|_{0} \leqslant(2 \mu)^{-1 / 2}\left\|p_{T}\right\|_{0},
$$

where $C_{\Omega}>0$ is the inf-sup constant. Thus, given that $\boldsymbol{u} \in \boldsymbol{H}_{\Gamma_{u, 0}}^{1}$ the bound (2.7) ensures that

$$
\begin{aligned}
\mathscr{B}\left(\boldsymbol{u}, p_{T}, p_{F} ;-\boldsymbol{v}, 0,0\right) & \geqslant C_{\Omega}(2 \mu)^{-1}\left\|p_{T}\right\|_{0}^{2}-(2 \mu)^{1 / 2}\|\nabla \boldsymbol{u}\|_{0}(2 \mu)^{1 / 2}\|\nabla \boldsymbol{v}\|_{0}, \\
& \geqslant C_{\Omega}(2 \mu)^{-1}\left\|p_{T}\right\|_{0}^{2}-(2 \mu)^{1 / 2}\|\nabla \boldsymbol{u}\|_{0}(2 \mu)^{-1 / 2}\left\|p_{T}\right\|_{0}, \\
& \geqslant\left(C_{\Omega}-\frac{1}{\varepsilon}\right)(2 \mu)^{-1}\left\|p_{T}\right\|_{0}^{2}-\varepsilon(2 \mu)\|\nabla \boldsymbol{u}\|_{0}^{2},
\end{aligned}
$$

for all $\varepsilon>0$. The coercivity bound (2.5) then leads to the following estimate

$$
\mathscr{B}\left(\boldsymbol{u}, p_{T}, p_{F} ; \boldsymbol{u},-p_{T},-p_{F}\right) \geqslant C_{K} 2 \mu\|\nabla \boldsymbol{u}\|_{0}^{2}+\frac{1}{\lambda}\left\|p_{T}\right\|_{0}^{2}+\frac{2 \alpha^{2}}{\lambda}\left\|p_{F}\right\|_{0}^{2}+\left\|\kappa \nabla p_{F}\right\|_{0}^{2}-2 \frac{\alpha}{\lambda}\left(p_{T}, p_{F}\right),
$$

where $C_{K}$ is the Korn constant.

Next, looking at the problematic product term, the Cauchy-Schwarz inequality and the arithmeticgeometric mean inequality imply that

$$
2 \frac{\alpha}{\lambda}\left(p_{T}, p_{F}\right) \leqslant \frac{1}{\varepsilon_{1} \lambda}\left\|p_{T}\right\|_{0}^{2}+\varepsilon_{1} \frac{\alpha^{2}}{\lambda}\left\|p_{F}\right\|_{0}^{2},
$$


for some $\varepsilon_{1}>0$. Introducing a parameter $\delta$ and combining (2.8), (2.9) and (2.10) then gives

$$
\begin{aligned}
\mathscr{B}\left(\boldsymbol{u}, p_{T}, p_{F} ; \boldsymbol{u}-\delta \boldsymbol{v},-p_{T},-p_{F}\right)= & \mathscr{B}\left(\boldsymbol{u}, p_{T}, p_{F} ; \boldsymbol{u},-p_{T},-p_{F}\right)+\boldsymbol{\delta} \mathscr{B}\left(\boldsymbol{u}, p_{T}, p_{F} ;-\boldsymbol{v}, 0,0\right) \\
\geqslant & C_{K} 2 \mu\|\nabla \boldsymbol{u}\|_{0}^{2}+\frac{1-\varepsilon_{1}}{\lambda}\left\|p_{T}\right\|_{0}^{2}+\left(2-\frac{1}{\varepsilon_{1}}\right) \frac{\alpha^{2}}{\lambda}\left\|p_{F}\right\|_{0}^{2} \\
& +\left\|\kappa^{\frac{1}{2}} \nabla p_{F}\right\|_{0}^{2}+\delta\left(C_{\Omega}-\frac{1}{\varepsilon}\right) \frac{1}{2 \mu}\left\|p_{T}\right\|_{0}^{2}-\delta \varepsilon(2 \mu)\|\nabla \boldsymbol{u}\|_{0}^{2}, \\
\geqslant & \left(C_{K}-\delta \varepsilon\right) 2 \mu\|\nabla \boldsymbol{u}\|_{0}^{2}+\left(2-\frac{1}{\varepsilon_{1}}\right) \frac{\alpha^{2}}{\lambda}\left\|p_{F}\right\|_{0}^{2} \\
& +\left\|\kappa^{\frac{1}{2}} \nabla p_{F}\right\|_{0}^{2}+\left(\frac{1-\varepsilon_{1}}{\lambda}+\delta\left(C_{\Omega}-\frac{1}{\varepsilon}\right)(2 \mu)^{-1}\right)\left\|p_{T}\right\|_{0}^{2} .
\end{aligned}
$$

Finally, the specific choice of parameters $\varepsilon=2 / C_{\Omega}, \delta=C_{\Omega} C_{K} / 4, \varepsilon_{1}=3 / 4$ together with $\boldsymbol{v}:=\boldsymbol{u}-\delta \boldsymbol{v}$, $q_{T}:=-p_{T}$ and $q_{F}=-p_{F}$, leads to the desired lower bound

$$
\mathscr{B}\left(\boldsymbol{u}, p_{T}, p_{F} ; \boldsymbol{u}-\delta \boldsymbol{v},-p_{T},-p_{F}\right) \geqslant \min \left\{\frac{1}{4}, \frac{C_{K}}{2}, \frac{C_{K} C_{\Omega}^{2}}{8}\right\}\|\|\left(\boldsymbol{u}, p_{T}, p_{F}\right)\|\|^{2} .
$$

To establish the upper bound we note that

$$
\begin{aligned}
2 \mu\|\nabla \boldsymbol{u}-\delta \nabla \boldsymbol{v}\|_{0}^{2} & \leqslant 2 \cdot 2 \mu\|\nabla \boldsymbol{u}\|_{0}^{2}+2 \delta^{2} \cdot 2 \mu\|\nabla \boldsymbol{v}\|_{0}^{2} \\
& \leqslant 2(2 \mu)\|\nabla \boldsymbol{u}\|_{0}^{2}+2 \delta^{2} \cdot(2 \mu)^{-1}\left\|p_{T}\right\|_{0}^{2}
\end{aligned}
$$

which gives the estimate

$$
\begin{aligned}
\left\|\mid\left(\boldsymbol{u}-\delta \boldsymbol{v},-p_{T},-p_{F}\right)\right\|^{2} & =2 \mu\|\nabla \boldsymbol{u}-\delta \nabla \boldsymbol{v}\|_{0}^{2}+(2 \mu)^{-1}\left\|p_{T}\right\|_{0}^{2}+\lambda^{-1}\left\|p_{T}\right\|_{0}^{2} \\
& +\frac{\alpha^{2}}{\lambda}\left\|p_{F}\right\|_{0}^{2}+\left\|\kappa^{1 / 2} \nabla p_{F}\right\|_{0}^{2} \\
& \leqslant\left(2+\frac{C_{K}^{2} C_{\Omega}^{2}}{8}\right)\left\|\left(\boldsymbol{u}, p_{T}, p_{F}\right)\right\|^{2}
\end{aligned}
$$

Note that the constants in (2.11) and (2.12) are independent of the Lamé coefficients, the modified hydraulic conductivity and the Biot-Willis parameter.

To construct a finite element approximation of (2.1), we recall that $\left\{\mathscr{T}_{h}\right\}$ denotes a family of shape regular rectangular/triangular meshes of $\bar{\Omega}$ into rectangles/triangles $K$ of diameter $h_{K}$. We let $\mathscr{E}_{h}$ be the the set of all edges of $\mathscr{T}_{h}, h_{E}$ be the length of the edge $E \in \mathscr{E}_{h}$ and we define finite-dimensional subsets $\boldsymbol{X}_{\boldsymbol{u}}^{h} \subset \boldsymbol{H}_{\Gamma_{\boldsymbol{u}}}^{1}, \boldsymbol{X}_{\boldsymbol{u}, 0}^{h} \subset \boldsymbol{H}_{\Gamma_{u, 0}}^{1}, M^{h} \subset M, X_{p}^{h} \subset H_{\Gamma_{p}}^{1}$ and $X_{p, 0}^{h} \subset H_{\Gamma_{p, 0}}^{1}$. The discrete weak formulation is then given by: find $\left(\boldsymbol{u}_{h}, p_{T, h}, p_{F, h}\right) \in \boldsymbol{X}_{\boldsymbol{u}}^{h} \times M^{h} \times X_{p}^{h}$ such that

$$
\begin{array}{rlrl}
a\left(\boldsymbol{u}_{h}, \boldsymbol{v}\right)+b_{1}\left(\boldsymbol{v}, p_{T, h}\right) & =f(\boldsymbol{v}) & & \forall \boldsymbol{v} \in \boldsymbol{X}_{\boldsymbol{u}, 0}^{h}, \\
b_{1}\left(\boldsymbol{u}_{h}, q_{T}\right)-c\left(p_{T, h}, q_{T}\right)+b_{2}\left(p_{F, h}, q_{T}\right) & =0 & \forall q_{T} \in M^{h}, \\
b_{2}\left(q_{F}, p_{T, h}\right)-d\left(p_{F, h}, q_{F}\right) & =g\left(q_{F}\right) & & \forall q_{F} \in X_{p, 0}^{h} .
\end{array}
$$


Analogously to (2.3), the discrete formulation can also be written as: find $\left(\boldsymbol{u}_{h}, p_{T, h}, p_{F, h}\right) \in \boldsymbol{X}_{\boldsymbol{u}}^{h} \times M^{h} \times X_{p}^{h}$ such that

$$
\mathscr{B}\left(\boldsymbol{u}_{h}, p_{T, h}, p_{F, h} ; \boldsymbol{v}, q_{T}, q_{F}\right)=f(\boldsymbol{v})+g\left(p_{F}\right) \quad \forall\left(\boldsymbol{v}, q_{T}, q_{F}\right) \in \boldsymbol{X}_{\boldsymbol{u}, 0}^{h} \times M^{h} \times X_{p, 0}^{h}
$$

The solution spaces $\boldsymbol{X}_{\boldsymbol{u}}^{h}$ and $X_{p}^{h}$ are defined as follows:

$$
\boldsymbol{X}_{\boldsymbol{u}}^{h}=\left\{\boldsymbol{u} \mid \boldsymbol{u}=\sum_{j=1}^{n_{u}} a_{j} \boldsymbol{\phi}_{j}+\sum_{j=n_{u}+1}^{n_{u}+n_{\partial_{u}}} a_{j} \boldsymbol{\phi}_{j}\right\}, X_{p}^{h}=\left\{p \mid p=\sum_{j=1}^{n_{p}} b_{j} \phi_{j}+\sum_{j=n_{p}+1}^{n_{p}+n_{\partial_{p}}} b_{j} \boldsymbol{\phi}_{j}\right\}
$$

where $a_{j}, b_{j} \in \mathbb{R},\left\{\boldsymbol{\phi}_{j}\right\}_{j=1}^{n_{u}}$ are vector valued basis functions that $\operatorname{span} \boldsymbol{X}_{\boldsymbol{u}, 0}^{h}$ and $\left\{\boldsymbol{\phi}_{j}\right\}_{j=1}^{n_{p}}$ are scalar valued basis functions that $\operatorname{span} X_{p, 0}^{h}$. The additional coefficients $\left\{a_{j}\right\}_{j=n_{u}+1}^{n_{u}+n_{\partial_{u}}}$ and $\left\{b_{j}\right\}_{j=n_{p}+1}^{n_{p}+n_{\partial_{p}}}$ are associated with Lagrange interpolation of the boundary data $\boldsymbol{g}$ on $\Gamma_{\boldsymbol{u}}$ and the boundary data $h$ on $\Gamma_{p}$, respectively. The finite-dimensional spaces $\boldsymbol{X}_{\boldsymbol{u}, 0}^{h}, M^{h}$ and $X_{p, 0}^{h}$ are related to $\left\{\mathscr{T}_{h}\right\}$.

The stability of the discrete weak formulation (2.14) follows directly from Theorem 2.1 if and only if the inf-sup condition (2.6) holds uniformly for the spaces $\boldsymbol{X}_{\boldsymbol{u}, 0}^{h}$ and $M^{h}$ : that is, for mixed approximation methods that satisfy

$$
\inf _{0 \neq q_{h} \in M^{h}} \sup _{0 \neq \boldsymbol{v}_{h} \in \boldsymbol{X}_{\boldsymbol{u}, 0}^{h}} \frac{\left|b_{1}\left(\boldsymbol{v}_{h}, q_{h}\right)\right|}{\left\|\nabla \boldsymbol{v}_{h}\right\|_{0}\left\|q_{h}\right\|_{0}} \geqslant \gamma
$$

where $\gamma$ is bounded away from zero independently of $h$.

While the analysis in the next section is applicable to any inf-sup stable approximation pair, the final sections of the paper will focus on the lowest-order conforming Taylor-Hood methods ${ }^{1}$ :

- $\boldsymbol{Q}_{2}-Q_{1}-Q_{2}$ (continuous biquadratic approximation of the two components of the displacement together with a continuous bilinear approximation of the total pressure field and a continuous biquadratic approximation of the fluid pressure) on a grid of rectangular elements.

- $\boldsymbol{P}_{2}-P_{1}-P_{2}$ (continuous quadratic approximation of the two components of the displacement together with a continuous linear approximation of the total pressure field and a continuous quadratic approximation of the fluid pressure) on a mesh of triangular elements.

REMARK 2.1 Stabilized lowest-order finite element approximation for a conventional three-field poroelasticity model involving displacement, fluid flux and fluid pressure has been studied by Berger et al. (2015) and in recent work by Frigo et al. (2020). In our formulation, in place of $\boldsymbol{Q}_{2}-Q_{1}-Q_{2} / \boldsymbol{P}_{2}-P_{1}-P_{2}$, we could use the lowest-order finite element $\boldsymbol{Q}_{1}-Q_{0}-Q_{1} / \boldsymbol{P}_{1}-P_{0}-P_{1}$ approximation triplet. Then to stabilize the finite element approximations of the displacement and total pressure, we simply need to add $-(2 \mu)^{-1}\left(\llbracket p_{T, h} \rrbracket, \llbracket q_{T} \rrbracket\right)$ in (2.13b) within macroelements (see Khan et al. (2018); Kechkar \& Silvester (1992) for details).

\footnotetext{
${ }^{1}$ These strategies are also inf-sup stable in a three-dimensional setting.
} 


\section{A posteriori error estimation}

\subsection{The residual error estimator}

We start by defining some important parameters for the analysis

$$
\begin{aligned}
& \rho_{1, K}=h_{K}(2 \mu)^{-\frac{1}{2}} / 2, \rho_{1, E}=h_{E}(2 \mu)^{-1} / 2, \rho_{d}=\min \left\{1 /\left(\lambda^{-1}+(2 \mu)^{-1}\right), \alpha^{-2} \lambda\right\}, \\
& \rho_{2, K}=\min \left\{h_{K} \kappa^{-1 / 2} / 2, \lambda^{1 / 2} \alpha^{-1}\right\}, \quad \rho_{2, E}=h_{E} \kappa_{E}^{-1} / 2,
\end{aligned}
$$

with $\kappa_{E}=\max \left\{\kappa_{K}, \kappa_{K^{\prime}}\right\}$, where $E$ is an interior edge that is shared by two elements $K$ and $K^{\prime}$, and $\kappa_{E}=\kappa_{K}$, if $E \in \partial K \cap \partial \Omega$. Next, we define a local error indicator $\eta_{K}$ for each element $K \in \mathscr{T}_{h}$. The square of this local error indicator is the sum of terms, $\eta_{K}^{2}=\eta_{R_{K}}^{2}+\eta_{E_{K}}^{2}+\eta_{J_{K}}^{2}$, with

$$
\begin{aligned}
& \eta_{R_{K}}^{2}=\rho_{1, K}^{2}\left\|\boldsymbol{R}_{K}\right\|_{0, K}^{2}+\rho_{2, K}^{2}\left\|R_{K}\right\|_{0, K}^{2}, \quad \eta_{J_{K}}^{2}=\rho_{d}\left\|\mathscr{R}_{K}\right\|_{0, K}^{2}, \\
& \eta_{E_{K}}^{2}=\sum_{E \in \partial K} \rho_{1, E}\left\|\boldsymbol{R}_{E}\right\|_{0, E}^{2}+\sum_{E \in \partial K} \rho_{2, E}\left\|R_{E}\right\|_{0, E}^{2}
\end{aligned}
$$

where the three element residuals are given by

$$
\begin{aligned}
& \boldsymbol{R}_{K}=\left.\left\{\boldsymbol{f}_{h}+\nabla \cdot\left(2 \mu \boldsymbol{\varepsilon}\left(\boldsymbol{u}_{h}\right)\right)-\nabla p_{T, h}\right\}\right|_{K}, \\
& \mathscr{R}_{K}=\left.\left\{\nabla \cdot \boldsymbol{u}_{h}+\frac{1}{\lambda} p_{T, h}-\frac{\alpha}{\lambda} p_{F, h}\right\}\right|_{K}, \\
& R_{K}=\left.\left\{g_{h}-\frac{\alpha}{\lambda}\left(p_{T, h}-2 \alpha p_{F, h}\right)-\nabla \cdot\left(\kappa \nabla p_{F, h}\right)\right\}\right|_{K}
\end{aligned}
$$

and the edge residual is associated with the normal stress jump, so that

$$
\begin{aligned}
& \boldsymbol{R}_{E}= \begin{cases}\frac{1}{2} \llbracket\left(p_{T, h} \boldsymbol{I}-2 \mu \boldsymbol{\varepsilon}\left(\boldsymbol{u}_{h}\right)\right) \boldsymbol{n} \rrbracket_{E} & E \in \mathscr{E}_{h} \backslash \Gamma, \\
\left(\left(p_{T, h} \boldsymbol{I}-2 \mu \boldsymbol{\varepsilon}\left(\boldsymbol{u}_{h}\right)\right) \boldsymbol{n}\right)_{E} & E \in \mathscr{E}_{h} \cap \Gamma_{p}, \\
0 & E \in \mathscr{E}_{h} \cap \Gamma_{\boldsymbol{u}} .\end{cases} \\
& \boldsymbol{R}_{E}= \begin{cases}\frac{1}{2} \llbracket\left(\kappa \nabla p_{F, h}\right) \cdot \boldsymbol{n} \rrbracket_{E} & E \in \mathscr{E}_{h} \backslash \Gamma, \\
\left(\left(\kappa \nabla p_{F, h}\right) \cdot \boldsymbol{n}\right)_{E} & E \in \mathscr{E}_{h} \cap \Gamma_{\boldsymbol{u}}, \\
0 & E \in \mathscr{E}_{h} \cap \Gamma_{p} .\end{cases}
\end{aligned}
$$

Let $\boldsymbol{f}_{h}$ and $g_{h}$ denote piecewise polynomial approximations of $\boldsymbol{f}$ and $g$, respectively, that are possibly discontinuous across element edges. We associate these functions with the data oscillation term

$$
\Theta_{K}^{2}=\rho_{1, K}^{2}\left\|\boldsymbol{f}-\boldsymbol{f}_{h}\right\|_{0, K}^{2}+\rho_{2, K}^{2}\left\|g-g_{h}\right\|_{0, K}^{2}
$$

The residual error estimator and data oscillation error are then defined as follows:

$$
\eta=\left(\sum_{K \in \mathscr{T}_{h}} \eta_{K}^{2}\right)^{1 / 2} \text { and } \Theta=\left(\sum_{K \in \mathscr{T}_{h}} \Theta_{K}^{2}\right)^{1 / 2}
$$

REMARK 3.1 Looking at the definition (3.1), and recalling that the conductivity $\kappa$ has been scaled by the time step $k_{t}$, we see that there are two terms $\rho_{2, K}$ and $\rho_{2, E}$ that potentially blow up in the limit $k_{t} \rightarrow 0$. The residual estimator is robust in the small time step limit nevertheless: first, $\lim _{k_{t} \rightarrow 0} \rho_{2, K}=\lambda^{1 / 2} \alpha^{-1}$ so the first contribution does not blow up. Second, while $\rho_{2, E}$ can blow up, the term $\rho_{2, E}\left\|R_{E}\right\|_{0, E}^{2}=C k_{t}$ so the edge estimator $\eta_{E_{K}}$ remains bounded when $k_{t} \rightarrow 0$. 
The reliability and efficiency of the residual estimator may be readily established using standard arguments involving quasi-interpolation estimates (Clément, 1975). A formal statement is given in the next two theorems. We note that these estimates hold for any conforming mixed approximation: the inf-sup stability bound (2.15) is not used in the proofs. ${ }^{2}$

THEOREM 3.1 (Reliability estimate) Suppose that $\left(\boldsymbol{u}, p_{T}, p_{F}\right)$ is the weak solution satisfying (2.1) and that $\left(\boldsymbol{u}_{h}, p_{T, h}, p_{F, h}\right) \in \boldsymbol{X}_{\boldsymbol{u}}^{h} \times M^{h} \times X_{p}^{h}$ is a conforming mixed approximation. Defining $\eta$ and $\Theta$ to be the error estimator and the data oscillation term in (3.10), we have an upper bound on the approximation error,

$$
\left\|\left(\boldsymbol{u}-\boldsymbol{u}_{h}, p_{T}-p_{T, h}, p_{F}-p_{F, h}\right)\right\| \mid \lesssim \eta+\Theta
$$

Proof. See Appendix A.

THEOREM 3.2 (Efficiency estimate) Suppose that $\left(\boldsymbol{u}, p_{T}, p_{F}\right)$ is the weak solution satisfying (2.1) and that $\left(\boldsymbol{u}_{h}, p_{T, h}, p_{F, h}\right) \in \boldsymbol{X}_{\boldsymbol{u}}^{h} \times M^{h} \times X_{p}^{h}$ is a conforming mixed approximation. Defining $\eta$ and $\Theta$ to be the error estimator and the data oscillation term in (3.10), we have a lower bound on the approximation error,

$$
\eta \lesssim||\left|\left(\boldsymbol{u}-\boldsymbol{u}_{h}, p_{T}-p_{T, h}, p_{F}-p_{F, h}\right) \|\right|+\Theta
$$

Proof. See Appendix A.

The reliability and efficiency of the simple residual error estimator $\eta$ provides the motivation for the introduction of equivalent local problem estimators that give a more accurate measurement of the actual error. The actual improvement in accuracy is quantified in the numerical results presented later. Two alternative local problem estimators will be considered here. Working in the framework established by Verfürth (2013), these local estimators can be shown to be robust and efficient with respect to the underlying problem parameters.

\subsection{Energy-based local error estimator}

The Biot problem error estimator

$$
\eta_{\mathscr{B}}=\sqrt{\sum_{K \in \mathscr{T}_{h}} \eta_{\mathscr{B}, K}^{2}}
$$

is assembled from estimates of element contributions to the energy error, given by

$$
\eta_{\mathscr{B}, K}^{2}=\left.\left|\|\left(\boldsymbol{e}_{\mathscr{B}, K}, \varepsilon_{\mathscr{B}, K}^{T}, \varepsilon_{\mathscr{B}, K}^{F}\right)\right|\right|_{K} ^{2},
$$

where

$$
\begin{aligned}
\left\|\left|\left(\boldsymbol{e}_{\mathscr{B}, K}, \varepsilon_{\mathscr{B}, K}^{T}, \varepsilon_{\mathscr{B}, K}^{F}\right) \|\right|_{K}^{2}=\right. & 2 \mu\left\|\boldsymbol{\varepsilon}\left(\boldsymbol{e}_{\mathscr{B}, K}\right)\right\|_{0, K}^{2}+(2 \mu)^{-1}\left\|\varepsilon_{\mathscr{B}, K}^{T}\right\|_{0, K}^{2}+\lambda^{-1}\left\|\varepsilon_{\mathscr{B}, K}^{T}\right\|_{0, K}^{2} \\
& +\alpha^{2}(\lambda)^{-1}\left\|\varepsilon_{\mathscr{B}, K}^{F}\right\|_{0, K}^{2}+\left\|\kappa^{1 / 2} \nabla \varepsilon_{\mathscr{B}, K}^{F}\right\|_{0, K}^{2} .
\end{aligned}
$$

To compute the local contributions, we solve the Biot's consolidation model problem on each element

\footnotetext{
${ }^{2}$ While the error estimation is precise in the case of an unstable mixed approximation, if the inf-sup constant in (2.15) is mesh dependent then the quality of the approximation is likely to be compromised when one is close to the incompressible limit.
} 
FIG. 1. $\boldsymbol{Q}_{3}(K)$ interpolation nodes for an interior element (left), edge element (middle) and a corner element (right).
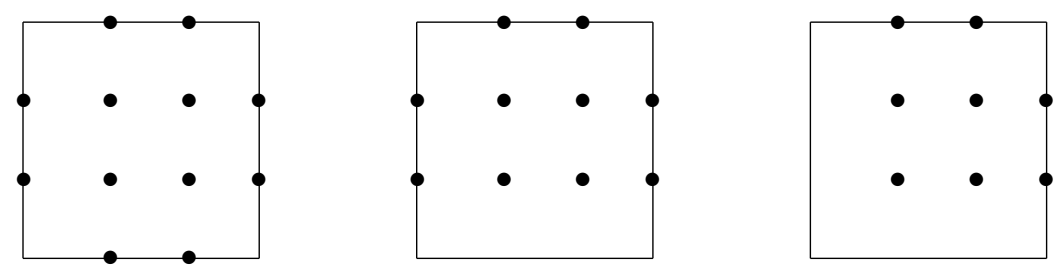

with selectively reduced correction spaces, see Liao \& Silvester (2012), $\mathscr{Q}_{K}=\boldsymbol{Q}_{3}(K) \times Q_{2}(K) \times Q_{3}(K)$ for $\boldsymbol{Q}_{2}-Q_{1}-Q_{2}$ mixed approximation (or $\mathscr{Q}_{K}=\boldsymbol{P}_{3}(K) \times P_{2}(K) \times P_{3}(K)$ for $\boldsymbol{P}_{2}-P_{1}-P_{2}$ mixed approximation): find $\left(\boldsymbol{e}_{\mathscr{B}, K}, \varepsilon_{\mathscr{B}, K}^{T}, \varepsilon_{\mathscr{B}, K}^{F}\right) \in \mathscr{Q}_{K}$, such that

$$
\begin{gathered}
2 \mu\left(\boldsymbol{\varepsilon}\left(\boldsymbol{e}_{\mathscr{B}, K}\right), \boldsymbol{\varepsilon}(\boldsymbol{v})\right)_{K}-\left(\varepsilon_{\mathscr{B}, K}, \nabla \cdot \boldsymbol{v}\right)_{K}=\boldsymbol{I}((v)), \quad \forall \boldsymbol{v} \in \boldsymbol{Q}_{3}(K) \\
-\left(\nabla \cdot \boldsymbol{e}_{\mathscr{B}, K}, q_{T}\right)_{K}-\frac{1}{\lambda}\left(\varepsilon_{\mathscr{B}, K}^{T}, q_{T}\right)_{K}+\frac{\alpha}{\lambda}\left(\varepsilon_{\mathscr{B}, K}^{F}, q_{T}\right)_{K}=-\left(\mathscr{R}_{K}, q_{T}\right)_{K}, \quad \forall q_{T} \in Q_{2}(K) . \\
\frac{\alpha}{\lambda}\left(\varepsilon_{\mathscr{B}, K}^{T}, q_{F}\right)_{K}-\frac{2 \alpha^{2}}{\lambda}\left(\varepsilon_{\mathscr{B}, K}^{F}, q_{F}\right)_{K}-\left(\kappa \nabla \varepsilon_{\mathscr{B}, K}^{F}, \nabla q_{F}\right)=I\left(q_{F}\right), \quad \forall q_{F} \in Q_{3}(K),
\end{gathered}
$$

where

$$
\boldsymbol{I}(\boldsymbol{v})=\left(\boldsymbol{R}_{K}, \boldsymbol{v}\right)_{K}-\sum_{E \in \partial K}\left\langle\boldsymbol{R}_{E}, \boldsymbol{v}\right\rangle_{E}, \quad \text { and } \quad I\left(q_{F}\right)=\left(R_{K}, q_{F}\right)_{K}-\sum_{E \in \partial K}\left\langle R_{E}, q_{F}\right\rangle_{E} .
$$

The following inf-sup estimate ensures local stability.

Lemma 3.1 (local inf-sup stability) Suppose that the space $Q_{3}(K)$ is defined with the degrees of freedom shown in Figure 1 then there exists a positive constant $\gamma_{L}$, satisfying

$$
\min _{0 \neq q_{h} \in \boldsymbol{Q}_{2}(K)} \max _{0 \neq \boldsymbol{v}_{h} \in \boldsymbol{Q}_{3}(K)} \frac{\left|\left(q_{h}, \nabla \cdot \boldsymbol{v}_{h}\right)\right|}{\left|\boldsymbol{v}_{h}\right|_{1}|| q_{h} \|_{0}} \geqslant \gamma_{L}
$$

for all $K \in \mathscr{T}_{h}$.

Proof. Adapting the strategy introduced in Verfürth (1989, Lemma 4.1), the result can be established by a simple eigenvalue computation on the reference element, see Liao \& Silvester (2012).

The condition (3.16) is a sufficient condition for the following local stability estimate.

LEMMA 3.2 (local $\mathscr{B}$ stability) For all $(\boldsymbol{w}, s, t) \in \mathscr{Q}_{K}$, we have that

$$
\max _{\left(\boldsymbol{v}, q_{T}, q_{F}\right) \in \mathscr{Q}_{K}} \frac{\mathscr{B}\left(\boldsymbol{w}, s, t ; \boldsymbol{v}, q_{T}, q_{F}\right)}{\|\|\left(\boldsymbol{v}, q_{T}, q_{F}\right) \mid \|_{K}} \gtrsim\|\mid(\boldsymbol{w}, s, t)\| \|_{K} .
$$

Upper and lower bounds of the local problem estimator $\eta_{\mathscr{B}, K}$ are identified in the next theorem.

THEOREM 3.3 In the case of $\mathscr{Q}_{K}$ mixed approximation of the Biot's consolidation problem (2.1), the local problem estimator $\eta_{\mathscr{B}, K}$ defined by (3.13)-(3.15) is equivalent to the local residual error estimator $\eta_{K}$ associated with (3.10), that is

$$
\eta_{\mathscr{B}, K} \lesssim \eta_{K} \lesssim \eta_{\mathscr{B}, K}, \quad \forall K \in \mathscr{T}_{h} .
$$


10 of 25

Proof. Using Lemma 3.2, we have

$$
\begin{aligned}
\eta_{\mathscr{B}, K} & =\|\|\left(\boldsymbol{e}_{\mathscr{B}, K}, \boldsymbol{\varepsilon}_{\mathscr{B}, K}^{T}, \boldsymbol{\varepsilon}_{\mathscr{B}, K}^{F}\right) \|_{K} \\
& \lesssim \max _{\left(\boldsymbol{v}, q_{T}, q_{F}\right) \in \mathscr{Q}_{K}} \frac{\mathscr{B}\left(\left(\boldsymbol{e}_{\mathscr{B}, K}, \boldsymbol{\varepsilon}_{\mathscr{B}, K}^{T}, \boldsymbol{\varepsilon}_{\mathscr{B}, K}^{F}\right) ;\left(\boldsymbol{v}, q_{T}, q_{F}\right)\right)}{\|\|\left(\boldsymbol{v}, q_{T}, q_{F}\right) \|_{K}}, \\
& \lesssim \max _{\left(\boldsymbol{v}, q_{T}, q_{F}\right) \in \mathscr{Q}_{K}} \frac{\boldsymbol{I}(\boldsymbol{v})-\left(q_{T}, \mathscr{R}_{K}\right)+I\left(q_{F}\right)}{\left\|\left(\boldsymbol{v}, q_{T}, q_{F}\right)\right\| \|_{K}} .
\end{aligned}
$$

Applying the Cauchy-Schwarz inequality and a trace theorem leads to

$$
\eta_{\mathscr{B}, K}=\|||\left(\boldsymbol{e}_{\mathscr{B}, K}, \varepsilon_{\mathscr{B}, K}^{T}, \varepsilon_{\mathscr{B}, K}^{F}\right)||_{K} \lesssim \eta_{K} .
$$

We will establish the upper bound by modifying the bubble-function technique that is used to establish the efficiency of the residual estimator in Appendix A. First we define $\left.\boldsymbol{w}\right|_{K}=\rho_{1, K}^{2} \boldsymbol{R}_{K} \chi_{K}$. From (A.5) and (3.15a) we conclude that

$$
\begin{aligned}
\rho_{1, K}^{2}\left\|\boldsymbol{R}_{K}\right\|_{0, K}^{2} & \lesssim\left(\boldsymbol{R}_{K}, \rho_{K}^{2} \chi_{K} \boldsymbol{R}_{K}\right)_{K} \\
& =\left(\boldsymbol{R}_{K}, \boldsymbol{w}\right)_{K}=2 \mu\left(\boldsymbol{\varepsilon}\left(\boldsymbol{e}_{\mathscr{B}, K}\right), \boldsymbol{\varepsilon}\left(\boldsymbol{w}_{K}\right)\right)_{K}-\left(\boldsymbol{\varepsilon}_{\mathscr{B}, K}, \nabla \cdot \boldsymbol{w}_{K}\right)_{K} .
\end{aligned}
$$

Using the Cauchy-Schwarz inequality in (3.19) gives

$$
\rho_{1, K}^{2}\left\|\boldsymbol{R}_{K}\right\|_{0, K}^{2} \lesssim\left(2 \mu\left\|\boldsymbol{\varepsilon}\left(\boldsymbol{e}_{\mathscr{B}, K}\right)\right\|_{0, K}^{2}+(2 \mu)^{-1}\left\|\boldsymbol{\varepsilon}_{\mathscr{B}, K}\right\|_{0, K}^{2}\right)^{1 / 2}(2 \mu)^{1 / 2}\left|\boldsymbol{w}_{K}\right|_{1, K} .
$$

Also, using (A.5), we have

$$
\rho_{1, K}^{2}\left\|\boldsymbol{R}_{K}\right\|_{0, K}^{2} \lesssim\left(2 \mu\left\|\boldsymbol{\varepsilon}\left(\boldsymbol{e}_{\mathscr{B}, K}\right)\right\|_{0, K}^{2}+(2 \mu)^{-1}\left|\boldsymbol{\varepsilon}_{\mathscr{B}, K}\right|_{0, K}^{2}\right)^{1 / 2} \eta_{R_{K}},
$$

which gives

$$
\rho_{1, K}^{2}\left\|\boldsymbol{R}_{K}\right\|_{0, K}^{2} \lesssim\left(2 \mu\left\|\boldsymbol{\varepsilon}\left(\boldsymbol{e}_{\mathscr{B}, K}\right)\right\|_{0, K}^{2}+(2 \mu)^{-1}\left|\boldsymbol{\varepsilon}_{\mathscr{B}, K}\right|_{0, K}^{2}\right) .
$$

Introducing $\left.w\right|_{K}=\rho_{2, K}^{2} R_{K} \chi_{K}$ and using (A.5) and (3.15c) then gives

$$
\begin{aligned}
\rho_{2, K}^{2}\left\|R_{K}\right\|_{0, K}^{2} & \lesssim\left(R_{K}, \rho_{2, K}^{2} \chi_{K} R_{K}\right)_{K} \\
& =\left(R_{K}, w\right)_{K} \\
& =\frac{\alpha}{\lambda}\left(\varepsilon_{\mathscr{B}, K}^{T}, w\right)_{K}-\frac{2 \alpha^{2}}{\lambda}\left(\varepsilon_{\mathscr{B}, K}^{F}, w\right)_{K}-\left(\kappa \nabla \varepsilon_{\mathscr{B}, K}^{F}, \nabla w\right) .
\end{aligned}
$$

Applying the Cauchy-Schwarz inequality in (3.21) and using (A.5) implies

$$
\rho_{2, K}^{2}\left\|R_{K}\right\|_{0, K}^{2} \lesssim\left(\lambda^{-1}\left\|\varepsilon_{\mathscr{B}, K}^{T}\right\|_{0, K}^{2}+\alpha^{2} \lambda^{-1}\left\|\varepsilon_{\mathscr{B}, K}^{F}\right\|_{0, K}^{2}+\left\|\kappa^{\frac{1}{2}} \nabla \varepsilon_{\mathscr{B}, K}^{F}\right\|_{0, K}^{2}\right) .
$$

Next, we introduce

$$
\Lambda=\rho_{E} \llbracket p_{T, h} \underline{I}-2 \mu \boldsymbol{\varepsilon}\left(\boldsymbol{u}_{h}\right) \rrbracket \chi_{E},
$$

where $\chi_{E}$ is an edge bubble function. Then, by (3.15a), it holds

$$
\begin{aligned}
\rho_{1, E}^{2}\left\|\boldsymbol{R}_{E}\right\|_{0, E}^{2} & \lesssim\left(\llbracket p_{T, h} \underline{I}-2 \mu \boldsymbol{\varepsilon}\left(\boldsymbol{u}_{h}\right) \rrbracket, \Lambda\right)_{E} \\
& =-2 \mu\left(\boldsymbol{\varepsilon}\left(\boldsymbol{e}_{\mathscr{B}, K}\right), \boldsymbol{\varepsilon}(\Lambda)\right)_{K}+\left(\boldsymbol{\varepsilon}_{\mathscr{B}, K}, \nabla \cdot \Lambda\right)_{K}+\left(\boldsymbol{R}_{K}, \Lambda\right)_{K} .
\end{aligned}
$$


Applying the Cauchy-Schwarz inequality in (3.23) and using (A.10) and (3.20) implies

$$
\rho_{1, E}^{2}\left\|\boldsymbol{R}_{E}\right\|_{0, E}^{2} \lesssim\left(2 \mu\left\|\boldsymbol{\varepsilon}\left(\boldsymbol{e}_{\mathscr{B}, K}\right)\right\|_{0, K}^{2}+(2 \mu)^{-1}\left\|\boldsymbol{\varepsilon}_{\mathscr{B}, K}\right\|_{0, K}^{2}\right)^{1 / 2}\left(\rho_{1, E}^{2}\left\|\boldsymbol{R}_{E}\right\|_{0, E}^{2}\right)^{1 / 2}
$$

which gives the bound

$$
\rho_{1, E}^{2}\left\|\boldsymbol{R}_{E}\right\|_{0, E}^{2} \lesssim\left(2 \mu\left\|\boldsymbol{\varepsilon}\left(\boldsymbol{e}_{\mathscr{B}, K}\right)\right\|_{0, K}^{2}+(2 \mu)^{-1}\left\|\boldsymbol{\varepsilon}_{\mathscr{B}, K}\right\|_{0, K}^{2}\right)
$$

Next, we introduce

$$
\tilde{\Lambda}=\rho_{2, E} \llbracket\left(\kappa \nabla p_{F, h}\right) \cdot \boldsymbol{n} \rrbracket \chi_{E},
$$

where $\chi_{E}$ is an edge bubble function. Then, by (3.15a), it holds, in particular,

$$
\begin{aligned}
\rho_{2, E}^{2}\left\|R_{E}\right\|_{0, E}^{2} & \left.\lesssim\left(\llbracket \kappa \nabla p_{F, h}\right) \cdot \boldsymbol{n} \rrbracket, \tilde{\Lambda}\right)_{E} \\
& =-\frac{\alpha}{\lambda}\left(\varepsilon_{\mathscr{B}, K}^{T}, \tilde{\Lambda}\right)_{K}+\frac{2 \alpha^{2}}{\lambda}\left(\varepsilon_{\mathscr{B}, K}^{F}, \tilde{\Lambda}\right)_{K}+\left(\kappa \nabla \varepsilon_{\mathscr{B}, K}^{F}, \nabla \tilde{\Lambda}\right)+\left(R_{K}, \tilde{\Lambda}\right)_{K} .
\end{aligned}
$$

Applying the Cauchy-Schwarz inequality in (3.25) and using (A.10) and (3.20) implies

$$
\rho_{2, E}^{2}\left\|R_{E}\right\|_{0, E}^{2} \lesssim\left(\lambda^{-1}\left\|\varepsilon_{\mathscr{B}, K}^{T}\right\|_{0, K}^{2}+\alpha^{2} \lambda^{-1}\left\|\varepsilon_{\mathscr{B}, K}^{F}\right\|_{0, K}^{2}+\left\|\kappa^{\frac{1}{2}} \nabla \varepsilon_{\mathscr{B}, K}^{F}\right\|_{0, K}^{2}\right) .
$$

Since $\mathscr{R}_{K}=\nabla \cdot \boldsymbol{u}_{h}+\frac{1}{\lambda} p_{T, h}-\frac{\alpha}{\lambda} p_{F, h} \in Q_{2}(K)$, then from (3.15b), it holds, in particular,

$$
\begin{aligned}
& \left(\nabla \cdot \boldsymbol{e}_{\mathscr{B}, K}, \mathscr{R}_{K}\right)_{K}+\frac{1}{\lambda}\left(\varepsilon_{\mathscr{B}, K}^{T}, \mathscr{R}_{K}\right)_{K}-\frac{\alpha}{\lambda}\left(\varepsilon_{\mathscr{B}, K}^{F}, \mathscr{R}_{K}\right)_{K}=\left(\mathscr{R}_{K}, \mathscr{R}_{K}\right)_{K}, \\
& \left\|\mathscr{R}_{K}\right\|_{0, K} \lesssim\left\|\nabla \cdot \boldsymbol{e}_{\mathscr{B}, K}+\frac{1}{\lambda} \varepsilon_{\mathscr{B}, K}^{T}-\frac{\alpha}{\lambda} \varepsilon_{\mathscr{B}, K}^{F}\right\|_{0, K} .
\end{aligned}
$$

Finally, using the argument in Lemma A.4 gives

$$
\begin{aligned}
\eta_{J_{K}}^{2}=\rho_{d}\left\|\mathscr{R}_{K}\right\|_{0, K}^{2} & \lesssim \rho_{d}\left\|\nabla \cdot \boldsymbol{e}_{\mathscr{B}, K}+\frac{1}{\lambda} \varepsilon_{\mathscr{B}, K}^{T}-\frac{\alpha}{\lambda} \varepsilon_{\mathscr{B}, K}^{F}\right\|_{0, K}^{2} \\
& \lesssim \rho_{d}\left\|\nabla \cdot \boldsymbol{e}_{\mathscr{B}, K}\right\|_{0, K}^{2}+\frac{\rho_{d}}{\lambda^{2}}\left\|\varepsilon_{\mathscr{B}, K}^{T}\right\|_{0, K}^{2}+\frac{\rho_{d} \alpha^{2}}{\lambda^{2}}\left\|\varepsilon_{\mathscr{B}, K}^{F}\right\|_{0, K}^{2} \\
& \lesssim 2 \mu\left\|\varepsilon\left(\boldsymbol{e}_{\mathscr{B}, K}\right)\right\|_{0, K}^{2}+\frac{1}{\lambda}\left\|\varepsilon_{\mathscr{B}, K}^{T}\right\|_{0, K}^{2}+\frac{\alpha^{2}}{\lambda}\left\|\varepsilon_{\mathscr{B}, K}^{F}\right\|_{0, K}^{2} \lesssim \eta_{\mathscr{B}, K}^{2}
\end{aligned}
$$

Combining (3.20), (3.22), (3.24), (3.26) and (3.27) gives the desired upper bound.

REMARK 3.2 If we were to employ the stabilized $\boldsymbol{Q}_{1}-Q_{0}-Q_{1} / \boldsymbol{P}_{1}-P_{0}-P_{1}$ triplet instead of Taylor-Hood approximation then suitable correction spaces would be given by $\mathscr{Q}=\boldsymbol{Q}_{2}(K) \times Q_{1}(K) \times Q_{2}(K) / \boldsymbol{P}_{2}(K) \times$ $P_{1}(K) \times P_{2}(K)$.

\subsection{Poisson problem local error estimator}

The final estimator that we consider simplifies the local problem in (3.15) in a clever way. It also circumvents the issue of finding inf-sup stable correction spaces. Recalling the definition of $\rho_{d}$ from (3.1), the Poisson problem estimator

$$
\eta_{P}=\sqrt{\sum_{K \in \mathscr{T}_{h}} \eta_{P, K}^{2}}
$$


is assembled from local contributions given by

$$
\eta_{P, K}^{2}=2 \mu\left\|\nabla \boldsymbol{e}_{P, K}\right\|_{0, K}^{2}+\rho_{d}^{-1}\left\|\varepsilon_{P, K}^{T}\right\|_{0, K}^{2}+\alpha^{2}(\lambda)^{-1}\left\|\varepsilon_{P, K}^{F}\right\|_{0, K}^{2}+\left\|\kappa^{1 / 2} \nabla \varepsilon_{P, K}^{F}\right\|_{0, K}^{2},
$$

where $\left(\boldsymbol{e}_{P, K}, \varepsilon_{P, K}^{T}, \varepsilon_{P, K}^{F}\right) \in \mathscr{Q}_{K}$ solve the following set of decoupled problems on each element:

$$
\begin{aligned}
2 \mu\left(\nabla \boldsymbol{e}_{P, K}, \nabla \boldsymbol{v}\right)_{K} & =\boldsymbol{I}(\boldsymbol{v}), \quad \forall \boldsymbol{v} \in \boldsymbol{Q}_{3}(K), \\
\rho_{d}^{-1}\left(\varepsilon_{P, K}^{T}, q_{T}\right)_{K} & =\left(\mathscr{R}_{K}, q_{T}\right)_{K}, \quad \forall q \in Q_{2}(K), \\
-\frac{2 \alpha^{2}}{\lambda}\left(\varepsilon_{P, K}^{F}, q_{F}\right)_{K}-\left(\kappa \nabla \varepsilon_{P, K}^{F}, \nabla q_{F}\right) & =I\left(q_{F}\right), \quad \forall q_{F} \in Q_{3}(K) .
\end{aligned}
$$

In compact form, we can write

$$
\mathscr{B}_{P, K}\left(\left(\boldsymbol{e}_{P, K}, \varepsilon_{P, K}^{T}, \varepsilon_{P, K}^{F}\right) ;\left(\boldsymbol{v}, q_{T}, q_{F}\right)\right)=\boldsymbol{I}(\boldsymbol{v})-\left(\mathscr{R}_{K}, q_{T}\right)_{K}+I\left(q_{F}\right),
$$

where

$$
\begin{aligned}
\mathscr{B}_{P, K}\left(\left(\boldsymbol{e}_{P, K}, \varepsilon_{P, K}^{T}, \varepsilon_{P, K}^{F}\right) ;\left(\boldsymbol{v}, q_{T}, q_{F}\right)\right)= & 2 \mu\left(\nabla \boldsymbol{e}_{P, K}, \nabla \boldsymbol{v}\right)_{K}-\frac{1}{\rho_{d}}\left(\varepsilon_{P, K}^{T}, q_{T}\right)_{K} \\
& -\frac{2 \alpha^{2}}{\lambda}\left(\varepsilon_{P, K}^{F}, q_{F}\right)_{K}-\left(\kappa \nabla \varepsilon_{P, K}^{F}, \nabla q_{F}\right),
\end{aligned}
$$

and

$$
\boldsymbol{I}(\boldsymbol{v})=\left(\boldsymbol{R}_{K}, \boldsymbol{v}\right)_{K}-\sum_{E \in \partial K}\left\langle\boldsymbol{R}_{E}, \boldsymbol{v}\right\rangle_{E}, \quad \text { and } \quad I\left(q_{F}\right)=\left(R_{K}, q_{F}\right)_{K}-\sum_{E \in \partial K}\left\langle R_{E}, q_{F}\right\rangle_{E} .
$$

The uncoupled formulation (3.29) is attractive from a computational perspective. First, since $\varepsilon_{P, K}^{T} \in$ $Q_{2}(K)$ it immediately holds that $\varepsilon_{P, K}^{T}=\rho_{d} \mathscr{R}_{K}=\rho_{d}\left(\nabla \cdot \boldsymbol{u}_{h}+\lambda^{-1} p_{T, h}-\alpha \lambda^{-1} p_{F, h}\right)$ so (3.28) simplifies to the following explicit calculation

$$
\eta_{P, K}^{2}=2 \mu\left\|\nabla \boldsymbol{e}_{P, K}\right\|_{0, K}^{2}+\rho_{d}\left\|\nabla \cdot \boldsymbol{u}_{h}+\lambda^{-1} p_{T, h}-\alpha \lambda^{-1} p_{F, h}\right\|_{0, K}^{2}+\alpha^{2} \lambda^{-1}\left\|\varepsilon_{P, K}^{F}\right\|_{0, K}^{2}+\left\|\kappa^{1 / 2} \nabla \varepsilon_{P, K}^{F}\right\|_{0, K}^{2}
$$

A second attractive feature is that the stability of the local problem (3.29) is guaranteed-there is no need to construct compatible correction spaces. In next theorem, we derive an equivalence result for the Poisson problem estimator.

THEOREM 3.4 The local problem estimator $\eta_{P, K}$ defined by (3.28) and (3.29) is equivalent to the energybased local error estimator $\eta_{\mathscr{B}, K}$ defined by (3.13),

$$
\eta_{\mathscr{P}, K} \lesssim \eta_{\mathscr{B}, K} \lesssim \eta_{\mathscr{P}, K}, \quad \forall K \in \mathscr{T}_{h} .
$$

Proof. By (3.15) and (3.29), for any $K \in \mathscr{T}_{h}$ and $\left(\boldsymbol{v}, q_{T}, q_{F}\right) \in \mathscr{Q}_{K}$, we have

$$
\begin{aligned}
2 \mu\left(\nabla \boldsymbol{e}_{P, K}, \nabla \boldsymbol{v}\right)_{K}-\rho_{d}^{-1}\left(\varepsilon_{P, K}^{T}, q_{T}\right)_{K}-\frac{2 \alpha^{2}}{\lambda}\left(\varepsilon_{P, K}^{F}, q_{F}\right)_{K}-\left(\kappa \nabla \varepsilon_{P, K}^{F}, \nabla q_{F}\right) \\
=\left(\boldsymbol{R}_{K} \boldsymbol{v}\right)-\sum_{E \in \partial K}\left(\boldsymbol{R}_{E}, \boldsymbol{v}\right)_{E}-\left(\mathscr{R}_{K}, q_{T}\right)+\left(R_{K}, q_{F}\right)_{K}-\sum_{E \in \partial K}\left\langle R_{E}, q_{F}\right\rangle_{E} \\
=2 \mu\left(\boldsymbol{\varepsilon}\left(\boldsymbol{e}_{\mathscr{B}, K}\right), \boldsymbol{\varepsilon}(\boldsymbol{v})\right)_{K}-\left(\boldsymbol{\varepsilon}_{\mathscr{B}, K}, \nabla \cdot \boldsymbol{v}\right)_{K}-\left(\nabla \cdot \boldsymbol{e}_{\mathscr{B}, K}, q_{T}\right)-\frac{1}{\lambda}\left(\boldsymbol{\varepsilon}_{\mathscr{B}, K}, q_{T}\right) \\
\quad+\frac{\alpha}{\lambda}\left(\varepsilon_{\mathscr{B}, K}^{T}, q_{F}\right)_{K}-\frac{2 \alpha^{2}}{\lambda}\left(\varepsilon_{\mathscr{B}, K}^{F}, q_{F}\right)_{K}-\left(\kappa \nabla \varepsilon_{\mathscr{B}, K}^{F}, \nabla q_{F}\right) \\
=\mathscr{B}\left(\left(\boldsymbol{e}_{\mathscr{B}, K}, \varepsilon_{\mathscr{B}, K}^{T}, \boldsymbol{\varepsilon}_{\mathscr{B}, K}^{F}\right) ;\left(\boldsymbol{v}, q_{T}, q_{F}\right)\right) .
\end{aligned}
$$


Using the local $\mathscr{B}$ stability bound from Lemma 3.2 gives

$$
\begin{aligned}
\|\|\left(\boldsymbol{e}_{\mathscr{B}, K}, \boldsymbol{\varepsilon}_{\mathscr{B}, K}^{T}, \boldsymbol{\varepsilon}_{\mathscr{B}, K}^{F}\right) \|_{K} & \lesssim \max _{\left(\boldsymbol{v}, q_{T}, q_{F}\right) \in \mathscr{Q}_{K}} \frac{\mathscr{B}\left(\left(\boldsymbol{e}_{\mathscr{B}, K}, \boldsymbol{\varepsilon}_{\mathscr{B}, K}^{T}, \boldsymbol{\varepsilon}_{\mathscr{B}, K}^{F}\right) ;\left(\boldsymbol{v}, q_{T}, q_{F}\right)\right)}{\left\|\left(\boldsymbol{v}, q_{T}, q_{F}\right)\right\|} \\
& =\max _{\left(\boldsymbol{v}, q_{T}, q_{F}\right) \in \mathscr{Q}_{K}} \frac{\mathscr{B}_{P, K}\left(\left(\boldsymbol{e}_{P, K}, \boldsymbol{\varepsilon}_{P, K}^{T}, \boldsymbol{\varepsilon}_{P, K}^{F}\right) ;\left(\boldsymbol{v}, q_{T}, q_{F}\right)\right)}{\left\|\left(\boldsymbol{v}, q_{T}, q_{F}\right)\right\|_{K}}
\end{aligned}
$$

Applying the Cauchy-Schwarz inequality implies that

$$
\left\|\left(\boldsymbol{e}_{\mathscr{B}, K}, \boldsymbol{\varepsilon}_{\mathscr{B}, K}^{T}, \varepsilon_{\mathscr{B}, K}^{F}\right)\right\|_{K} \lesssim \eta_{P, K}
$$

To establish the upper bound, we take $\boldsymbol{v} \in \boldsymbol{Q}_{3}(K)$. Using (3.15a) and (3.29a) then leads to

$$
\begin{aligned}
2 \mu\left(\nabla \boldsymbol{e}_{P, K}, \nabla \boldsymbol{v}\right)_{K} & =\left(\boldsymbol{R}_{K} \boldsymbol{v}\right)-\sum_{E \in \partial K}\left(\boldsymbol{R}_{E}, \boldsymbol{v}\right)_{E} \\
& =2 \mu\left(\boldsymbol{\varepsilon}\left(\boldsymbol{e}_{\mathscr{B}, K}\right), \boldsymbol{\varepsilon}(\boldsymbol{v})\right)_{K}-\left(\boldsymbol{\varepsilon}_{\mathscr{B}, K}, \nabla \cdot \boldsymbol{v}\right)_{K}
\end{aligned}
$$

From (3.15b) and (3.29b), for any $q_{T} \in Q_{2}(K)$, it holds

$$
\begin{aligned}
\rho_{d}^{-1}\left(\varepsilon_{P, K}^{T}, q_{T}\right)_{K} & =\left(\mathscr{R}_{K}, q_{T}\right)_{K} \\
& =\left(\nabla \cdot \boldsymbol{e}_{\mathscr{B}, K}, q_{T}\right)_{K}+\frac{1}{\lambda}\left(\varepsilon_{\mathscr{B}, K}^{T}, q_{T}\right)_{K}-\frac{\alpha}{\lambda}\left(\varepsilon_{\mathscr{B}, K}^{T}, q_{F}\right)_{K}
\end{aligned}
$$

By (3.15c) and (3.29c), for any $q_{F} \in Q_{3}(K)$ we have

$$
\begin{aligned}
-\frac{2 \alpha^{2}}{\lambda}\left(\varepsilon_{P, K}^{F}, q_{F}\right)_{K}-\left(\kappa \nabla \varepsilon_{P, K}^{F}, \nabla q_{F}\right) & =I\left(q_{F}\right) \\
& =\frac{\alpha}{\lambda}\left(\varepsilon_{\mathscr{B}, K}^{T}, q_{F}\right)_{K}-\frac{2 \alpha^{2}}{\lambda}\left(\varepsilon_{\mathscr{B}, K}^{F}, q_{F}\right)_{K}-\left(\kappa \nabla \varepsilon_{\mathscr{B}, K}^{F}, \nabla q_{F}\right)
\end{aligned}
$$

Using (3.33) gives

$$
\begin{aligned}
(2 \mu)^{1 / 2}\left\|\nabla \boldsymbol{e}_{P, K}\right\|_{0, K} & =\max _{v \in \boldsymbol{Q}_{3}(K)} \frac{2 \mu\left(\nabla \boldsymbol{e}_{P, K}, \nabla \boldsymbol{v}\right)}{(2 \mu)^{1 / 2}\|\nabla \boldsymbol{v}\|_{0, K}} \\
& =\max _{v \in \boldsymbol{Q}_{3}(K)} \frac{2 \mu\left(\boldsymbol{\varepsilon}\left(\boldsymbol{e}_{\mathscr{B}, K}\right), \boldsymbol{\varepsilon}(\boldsymbol{v})\right)_{K}-\left(\boldsymbol{\varepsilon}_{\mathscr{B}, K}, \nabla \cdot \boldsymbol{v}\right)_{K}}{(2 \mu)^{1 / 2}\|\boldsymbol{\varepsilon}(\boldsymbol{v})\|_{0, K}} .
\end{aligned}
$$

Applying the Cauchy-Schwarz inequality leads to

$$
(2 \mu)^{1 / 2}\left\|\nabla \boldsymbol{e}_{P, K}\right\|_{0, K} \lesssim(2 \mu)^{1 / 2}\left\|\boldsymbol{\varepsilon}\left(\boldsymbol{e}_{\mathscr{B}, K}\right)\right\|_{0, K}+(2 \mu)^{-1 / 2}\left\|\boldsymbol{\varepsilon}_{\mathscr{B}, K}^{T}\right\|_{0, K}
$$


Using (3.34), we have

$$
\begin{aligned}
\rho_{d}^{-1 / 2}\left\|\varepsilon_{P, K}^{T}\right\|_{0, K} & =\max _{q \in Q_{2}(K)} \frac{\rho_{d}^{-1}\left(\varepsilon_{P, K}^{T}, q\right)}{\rho_{d}^{-1 / 2}\|q\|_{0, K}} \\
& =\max _{q \in Q_{2}(K)} \frac{\left(\nabla \cdot \boldsymbol{e}_{\mathscr{B}, K}, q\right)_{K}+\frac{1}{\lambda}\left(\varepsilon_{\mathscr{B}, K}^{T}, q\right)_{K}-\frac{\alpha}{\lambda}\left(\varepsilon_{\mathscr{B}, K}^{F}, q\right)_{K}}{\rho_{d}^{-1 / 2}\|q\|_{0, K}} \\
& \leqslant \max _{q \in Q_{2}(K)} \frac{\rho_{d}^{1 / 2}\left\|\nabla \cdot \boldsymbol{e}_{\mathscr{B}, K}+\frac{1}{\lambda} \varepsilon_{\mathscr{B}, K}^{T}-\frac{\alpha}{\lambda} \varepsilon_{\mathscr{B}, K}^{F}\right\|_{0, K} \rho_{d}^{-1 / 2}\|q\|_{0, K}}{\rho_{d}^{-1 / 2}\|q\|_{0, K}} \\
& \leqslant \rho_{d}^{1 / 2}\left\|\nabla \cdot \boldsymbol{e}_{\mathscr{B}, K}+\frac{1}{\lambda} \varepsilon_{\mathscr{B}, K}-\frac{\alpha}{\lambda} \varepsilon_{\mathscr{B}, K}^{F}\right\|_{0, K} .
\end{aligned}
$$

By (3.27), it holds

$$
\begin{aligned}
\rho_{d}^{-1}\left\|\varepsilon_{P, K}^{T}\right\|_{0, K}^{2} & \leqslant \rho_{d}\left\|\nabla \cdot \boldsymbol{e}_{\mathscr{B}, K}+\frac{1}{\lambda} \varepsilon_{\mathscr{B}, K}-\frac{\alpha}{\lambda} \varepsilon_{\mathscr{B}, K}^{F}\right\|_{0, K}^{2} \\
& \lesssim(2 \mu)\left\|\boldsymbol{\varepsilon}\left(\boldsymbol{e}_{\mathscr{B}, K}\right)\right\|_{0, K}^{2}+(\lambda)^{-1}\left\|\varepsilon_{\mathscr{B}, K}^{T}\right\|_{0, K}^{2}+\alpha^{2}(\lambda)^{-1}\left\|\varepsilon_{\mathscr{B}, K}^{F}\right\|_{0, K}^{2} .
\end{aligned}
$$

Similarly, it is easily shown that

$$
\frac{\alpha^{2}}{\lambda}\left\|\varepsilon_{P, K}^{F}\right\|_{0, K}^{2}+\left\|\kappa^{1 / 2} \varepsilon_{P, K}^{F}\right\|_{0, K}^{2} \lesssim \frac{1}{\lambda}\left\|\varepsilon_{\mathscr{B}, K}^{T}\right\|_{0, K}^{2}+\frac{\alpha^{2}}{\lambda}\left\|\varepsilon_{\mathscr{B}, K}^{F}\right\|_{0, K}^{2}+\left\|\kappa^{1 / 2} \nabla \varepsilon_{\mathscr{B}, K}^{F}\right\|_{0, K}^{2}
$$

Combining (3.37), (3.39) and (3.40) gives the required upper bound.

REMARK 3.3 All the constants that arise in the proof of Theorem 3.3 and Theorem 3.4 are independent of $h_{E}, h_{K}, \mu, \lambda, \alpha$ and $\kappa$. This confirms our assertion that the proposed local error estimators $\eta_{\mathscr{B}, K}$ and $\eta_{P, K}$ are fully robust.

\section{Computational results}

Some numerical results are presented in this section. These support the theoretical results presented previously. More specifically, we discuss computational results for the residual estimator $\eta$ defined in Section 3.1 and the Poisson problem local estimator $\eta_{P}$ defined in Section 3.3. In our first test problem a three-field approximation is computed with $\boldsymbol{Q}_{2}-Q_{1}-Q_{2}$ square elements using functionality from the IFISS toolbox ${ }^{3}$ (see Elman et al. (2014) for a review). Note that, for a uniform mesh with $n$ degrees of freedom, we have that $\mathscr{O}\left(n^{-r}\right) \approx \mathscr{O}\left(h^{2 r}\right)$, where $r>0$. In our second test problem we use $\boldsymbol{P}_{2}-P_{1}-P_{2}$ triangular elements. We start from an initial mesh $\mathscr{T}_{0}$ and then run the iterative refinement loop

$$
\text { Solve } \rightarrow \text { Estimate } \rightarrow \text { Mark } \rightarrow \text { Refine }
$$

to generate a (nested) sequence of regular meshes $\left\{\mathscr{T}_{\ell}\right\}$ using functionality taken from the T-IFISS toolbox ${ }^{4}$; see Bespalov et al. (2020) for details. The ingredients are as follows.

\footnotetext{
${ }^{3}$ IFISS version 3.6 runs under MATLAB or Octave. It can be downloaded from http://www. manchester.ac.uk/ ifiss/download.html and is compatible with Windows, Linux and MacOS computers.

${ }^{4}$ The software can be downloaded from http://www. manchester.ac.uk/ifiss/tifiss.html.
} 
Solve : solve the problem (2.14) for each $\mathscr{T}_{\ell}$ to generate the finite element approximation $\left(\boldsymbol{u}_{h}, p_{T, h}, p_{F, h}\right)$.

Estimate: compute $\eta_{\ell}=\sum_{K \in \mathscr{T}_{h}} \eta_{K}$ or $\eta_{\ell}=\sum_{K \in \mathscr{T}_{h}} \eta_{P, K}$.

Mark : determine a minimal subset $\mathscr{M}_{\ell}$ of marked triangles such that $\sum_{K \in \mathscr{M}_{\ell}} \eta_{K}^{2} \geqslant \theta \eta_{\ell}^{2}$ (and similarly with $\eta_{P, K}$ ), where $\theta=0.5$ is the bulk parameter (Dörfler, 1996).

Refine : use the red-green-blue strategy (Verfürth, 2013) for mesh refinement. ${ }^{5}$

\subsection{Analytic solution}

The first problem is posed on a square domain $\Omega=(0,1) \times(0,1)$ with a zero essential boundary condition on $\partial \Omega=\Gamma$. The exact displacement vector $\boldsymbol{u}=\left(u_{1}, u_{2}\right)^{\top}$ and the exact fluid pressure $p_{F}$ are given by the following expressions

$$
\boldsymbol{u}=\left\{\begin{array}{l}
u_{1}=\pi \cos (\pi y) \sin ^{2}(\pi x) \sin (\pi y) \\
u_{2}=-\pi \cos (\pi x) \sin ^{2}(\pi y) \sin (\pi x)
\end{array} \quad p_{F}=x\left(1-x^{2}\right) y\left(1-y^{2}\right)\right.
$$

FIG. 2. Energy error $\|||\| \mid$ and estimated energy errors $\eta$ and $\eta_{P}$ computed using $\boldsymbol{Q}_{2}-Q_{1}-Q_{2}$ approximation for the first test problem with $\lambda=1$ (left) and $\lambda=10^{4}$ (right).
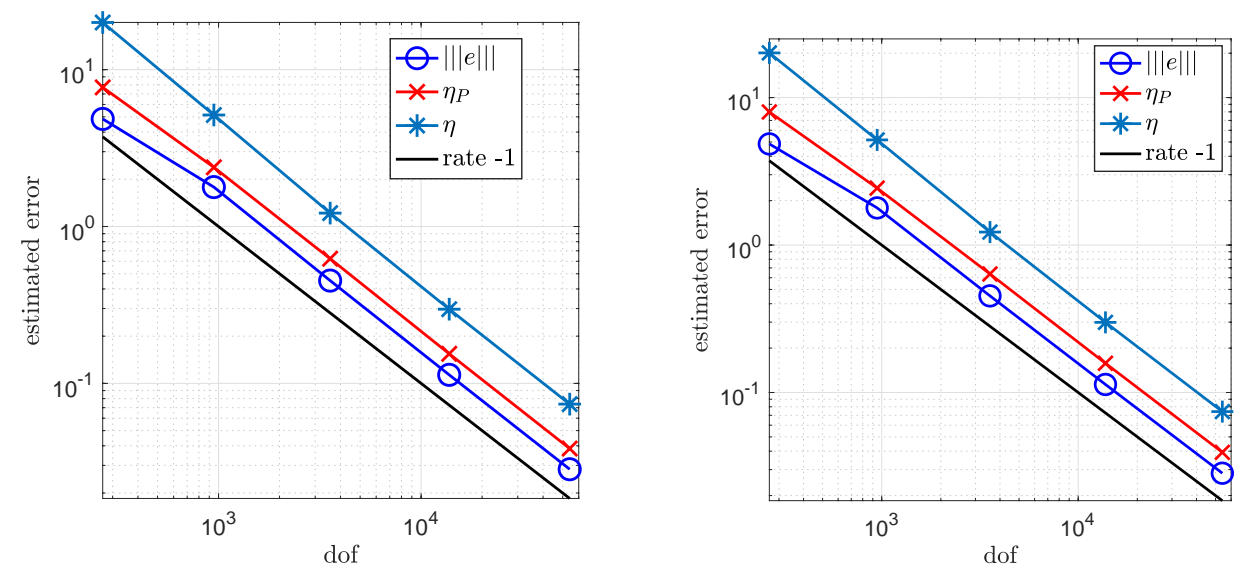

The convergence profile of the energy norm of the exact error $e=\|\|\left(\boldsymbol{u}-\boldsymbol{u}_{h}, p_{T}-p_{T, h}, p_{F}-p_{F, h}\right)\|\|$ and the estimates obtained with the alternative error estimation strategies are shown in Figure 2 . In this experiment, $\mu, \alpha$, and $\kappa$ are fixed and we choose two representative values of $\lambda$. In either case, we observe that the energy norm error decays to zero at the optimal rate $\mathscr{O}\left(n_{d o f}^{-1}\right)$ for a smooth solution. The fact that the convergence plots of the estimators $\eta$ and $\eta_{P}$ are parallel to the convergence plot of the exact error provides confirmation that both estimators are efficient and reliable. The plots also show that local problem estimator $\eta_{P}$ is a more accurate estimate of the error than $\eta$. This point is reinforced by the computed effectivity indices tabulated in Table 1 . These numbers confirm that the effectivity indices

${ }^{5}$ The refinement strategy built into T-IFISS version 1.2 is a variant of the newest vertex bisection (NVB) method. 
of the Poisson problem local estimator are close to unity for a range of different values of $\alpha$ and $\lambda$. Other computations (not reported here) show that the effectivity of both estimators is also completely insensitive to the value of $\kappa$ (we tested $\kappa=1$ and $\kappa=10^{-10}$ ). This provides confirmation that the estimators are robust with respect to all the underlying physical parameters.

\subsection{Singular solution}

A more realistic test of the error estimation strategies arises with a mixed boundary condition. To illustrate this, let us consider the square domain $\Omega=(-1,1) \times(-1,1)$ with $\Gamma_{\boldsymbol{u}}=\{\{-1\} \times[-1,1)\} \cup$ $\{[-1,1) \times\{-1\}\}$ and $\Gamma_{p}=\{\{1\} \times[-1,1]\} \cup\{[-1,1] \times\{1\}\}$. The parameters are fixed so that $\mu=1$, $\alpha=0.01$ and $\kappa=10^{-10}$. We then solve the problem with $\boldsymbol{f}=(1,1)^{\top}, g=1, \boldsymbol{g}=\mathbf{0}$ and $h=0$. Since the solution does not even have $H^{2}$ regularity-there are strong singularities at the two corners where the boundary condition changes - we anticipate that adaptive refinement will be needed if the optimal rate of convergence is to be realised.

Table 1. Effectivity indices computed using the residual and local Poisson problem estimators, for fixed $\mu=1$ and $\kappa=10^{-5}$ together with varying $\lambda, \alpha$ and $h$.

\begin{tabular}{crrrrrrr} 
& & \multicolumn{2}{c}{$\lambda=1$} & \multicolumn{2}{c}{$\lambda=10^{2}$} & \multicolumn{2}{c}{$\lambda=10^{4}$} \\
& $h$ & $\eta / e$ & $\eta_{P} / e$ & $\eta / e$ & $\eta_{P} / e$ & $\eta / e$ & $\eta_{P} / e$ \\
\cline { 2 - 8 }$\alpha=1$ & $1 / 4$ & 2.88 & 1.33 & 2.89 & 1.36 & 2.89 & 1.36 \\
& $1 / 8$ & 2.69 & 1.37 & 2.70 & 1.40 & 2.70 & 1.40 \\
& $1 / 16$ & 2.62 & 1.36 & 2.63 & 1.39 & 2.63 & 1.39 \\
& $1 / 32$ & 2.60 & 1.35 & 2.61 & 1.38 & 2.61 & 1.38 \\
\cline { 2 - 7 }$\alpha=10^{-2}$ & $1 / 4$ & 2.88 & 1.33 & 2.89 & 1.36 & 2.89 & 1.36 \\
& $1 / 8$ & 2.69 & 1.37 & 2.70 & 1.40 & 2.70 & 1.40 \\
& $1 / 16$ & 2.62 & 1.36 & 2.63 & 1.39 & 2.63 & 1.39 \\
$\alpha=10^{-4}$ & $1 / 32$ & 2.60 & 1.35 & 2.61 & 1.38 & 2.61 & 1.38 \\
\cline { 2 - 7 } & $1 / 4$ & 2.88 & 1.33 & 2.89 & 1.36 & 2.89 & 1.36 \\
& $1 / 8$ & 2.69 & 1.37 & 2.70 & 1.40 & 2.70 & 1.40 \\
& $1 / 16$ & 2.62 & 1.36 & 2.63 & 1.39 & 2.63 & 1.39 \\
& $1 / 32$ & 2.60 & 1.35 & 2.61 & 1.38 & 2.61 & 1.38
\end{tabular}

The results obtained using our adaptive refinement algorithm are compared with those obtained using uniform refinement in Figure 3. Looking at the error plots it is evident that, independent of $\lambda$, the convergence rate of $\eta_{P}$ is limited by the solution regularity when the grid is refined uniformly. We also see that both $\eta$ and $\eta_{P}$ converge to zero with the optimal rate $\mathscr{O}\left(n_{\text {dof }}^{-1}\right)$ when the grid is refined adaptively. The refinement pattern generated by the adaptive algorithm is slightly different in the two cases that we consider. While there is local refinement to resolve the strong singularities in both cases, there is additional local refinement to resolve the weak singularity that is generated at the corner $(-1,-1)$ in the (compressible) case $\lambda=1$. 
FIG. 3. Comparison of estimated energy errors $\eta$ and $\eta_{P}$ computed using $\boldsymbol{P}_{2}-P_{1}-P_{2}$ approximation for the second test problem with $\lambda=1$ (left) and $\lambda=10^{4}$ (right).
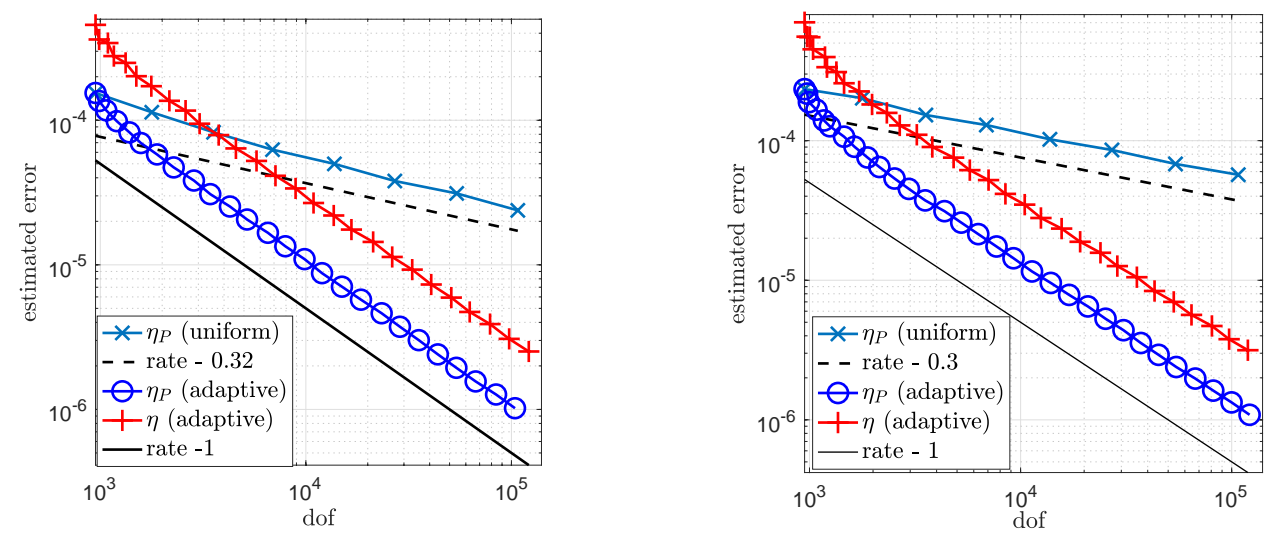

\section{Concluding remarks}

There are two important contributions in this paper. First, we have identified three parameter-robust error estimators for computing approximations of poroelasticity problems. We have shown that these estimators give reliable estimates of the approximation error, even when one is arbitrarily close to the incompressible limit. Second, we have identified a practical error estimation strategy based on solving three uncoupled Poisson problems that yields effectivity indices close to unity in all cases tested. Extending this work to enable the adaptive solution of poroelasticity problems with uncertain material parameters is ongoing research; see Khan \& Powell (2020). Ensuring robustness in the error estimation process is fundamentally important when solving problems with large variability in the measurement of such parameters.

\section{Funding}

Engineering and Physical Sciences Research Council (EP/P013317).

\section{REFERENCES}

Ahmed, E., Radu, F. A. \& Nordbotten, J. M. (2019) Adaptive poromechanics computations based on a posteriori error estimates for fully mixed formulations of Biot's consolidation model. Comput. Methods Appl. Mech. Engrg., 347, 264-294.

Ahmed, E., Nordbotten, J. M. \& Radu, F. A. (2020) Adaptive asynchronous time-stepping, stopping criteria, and a posteriori error estimates for fixed-stress iterative schemes for coupled poromechanics problems. Comput. Methods Appl. Mech. Engrg., 364, 112312.

Ainsworth, M. \& Oden, J. T. (2000) A Posteriori Error Estimation in Finite Element Analysis. New York: Wiley.

Berger, L., Bordas, R., Kay, D. \& TAVener, S. (2015) Stabilized lowest-order finite element approximation for linear three-field poroelasticity. SIAM J. Sci. Comput., 37, A2222-A2245.

Bespalov, A., Rocchi, L. \& Silvester, D. (2020) T-IFISS: a toolbox for adaptive FEM computation. Comput. Math. Appl. https://doi.org/10.1016/j.camwa.2020.03.005. 
Boffi, D., Brezzi, F. \& Fortin, M. (2013) Mixed Finite Element Methods and Applications. Heidelberg: Springer.

Chen, Y., Luo, Y. \& Feng, M. (2013) Analysis of a discontinuous Galerkin method for the Biot's consolidation problem. Appl. Math. Comput., 219, 9043-9056.

ClÉment, P. (1975) Approximation by finite element functions using local regularization. R.A.I.R.O. Anal. Numér., 2, 77-84.

Dörfler, W. (1996) A convergent adaptive algorithm for Poisson's equation. SIAM J. Numer. Anal., 33, 11061124.

Elman, H., Ramage, A. \& Silvester, D. (2014) IFISS: A computational laboratory for investigating incompressible flow problems. SIAM Rev., 56, 261-273.

ERn, A. \& MEunier, S. (2009) A posteriori error analysis of Euler-Galerkin approximations to coupled ellipticparabolic problems. ESAIM: M2AN, 43, 353-375.

Frigo, M., Castelletto, N., Ferronato, M. \& White, J. A. (2020) Efficient solvers for hybridized threefield mixed finite element coupled poromechanics. arXiv preprint https://arxiv. org/abs/2002.09603.

KeChKAR, N. \& Silvester, D. (1992) Analysis of locally stabilized mixed finite element methods for the Stokes problem. Math. Comput., 58, 1-10.

Khan, A., Powell, C. \& Silvester, D. (2017) Robust a posteriori error estimators for mixed approximation of nearly incompressible elasticity. arXiv preprint https: / / arxiv. org/abs/1710.03328.

Khan, A., Powell, C. \& Silvester, D. (2018) Robust error estimation for lowest-order approximation of nearly incompressible elasticity. arXiv preprint https://arxiv.org/abs/1801.04122.

Khan, A. \& Powell, C. (2020) Parameter-robust stochastic Galerkin mixed approximation for linear poroelasticity with uncertain inputs. arXiv preprint https://arxiv.org/abs/2003.06628.

Korsawe, J. \& Starke, G. (2005) A least-squares mixed finite element method for Biot's consolidation problem in porous media. SIAM J. Numer. Anal., 43, 318-339.

LeE, J. J., MARdAl, K.-A. \& Winther, R. (2017) Parameter-robust discretization and preconditioning of Biot's consolidation model. SIAM J. Sci. Comput., 39, A1-A24.

LI, Y. \& ZiKATANOV, L. T. (2019) Residual-based a posteriori error estimates of mixed methods in Biot's consolidation model. arXiv preprint https://arxiv.org/abs/1911.08692.

LiaO, Q. \& Silvester, D. (2012) A simple yet effective a posteriori error estimator for classical mixed approximation of Stokes equations. Appl. Numer. Math., 62, 1242-1256.

Murad, M. A., Thomée, V. \& Loula, A. F. (1996) Asymptotic behavior of semidiscrete finite-element approximations of Biot's consolidation problem. SIAM J. Numer. Anal., 33, 1065-1083.

Murad, M. A. \& LoulA, A. F. (1992) Improved accuracy in finite element analysis of Biot's consolidation problem. Comput. Methods Appl. Mech. Engrg., 95, 359-382.

Murad, M. A. \& Loula, A. F. (1994) On stability and convergence of finite element approximations of Biot's consolidation problem. Int. J. Numer. Meth. Engng., 37, 645-667.

Oyarzúa, R., Rhebergen, S. \& Manuel Solano, P. Z. (2019) Error analysis of a conforming and lockingfree four-field formulation in poroelasticity. $\mathrm{CI}^{2} \mathrm{MA}$ preprint 2019-31.

OyARZÚA, R. \& RUIZ-BAIER, R. (2016) Locking-free finite element methods for poroelasticity. SIAM J. Numer. Anal., 54, 2951-2973.

Phillips, P. J. \& WheEler, M. F. (2007a) A coupling of mixed and continuous Galerkin finite element methods for poroelasticity i: the continuous in time case. Comput. Geosci., 11, 131.

Phillips, P. J. \& Wheeler, M. F. (2007b) A coupling of mixed and continuous Galerkin finite element methods for poroelasticity ii: the discrete-in-time case. Comput. Geosci., 11, 145-158.

Phillips, P. J. \& WheEler, M. F. (2008) A coupling of mixed and discontinuous Galerkin finite-element methods for poroelasticity. Comput. Geosci., 12, 417-435.

REED, M. (1984) An investigation of numerical errors in the analysis of consolidation by finite elements. Int. J. Numer. Anal. Methods Geomech., 8, 243-257. 
Verfürth, R. (1989) A posteriori error estimators for the Stokes equations. Numer. Math., 55, 309-325.

VERfürth, R. (2013) A Posteriori Error Estimation Techniques for Finite Element Methods. Oxford: Oxford University Press.

Vermeer, P. \& Verruit, A. (1981) An accuracy condition for consolidation by finite elements. Int. J. Numer. Anal. Methods Geomech., 5, 1-14.

YI, S.-Y. (2013) A coupling of nonconforming and mixed finite element methods for Biot's consolidation model. Numer. Methods Partial Differential Equations, 29, 1749-1777.

ZIENkiEwICZ, O. \& ShIOMI, T. (1984) Dynamic behaviour of saturated porous media; the generalized Biot formulation and its numerical solution. Int. J. Numer. Anal. Methods Geomech., 8, 71-96. 


\section{Appendix A. Residual estimator error bounds}

The starting point for showing the reliability of the residual estimator introduced in Section 3.1 is the following lemma.

LemmA A.1 (Clément interpolation estimate) Given $\boldsymbol{v} \in \boldsymbol{H}_{\Gamma_{\boldsymbol{u}, 0}}^{1}$ and $q_{F} \in H_{p, 0}^{1}$, let $\boldsymbol{v}_{h} \in \boldsymbol{X}_{\boldsymbol{u}, 0}^{h}$ and $q_{F, h} \in$ $X_{p, 0}^{h}$ be the quasi-interpolant of $v$ and $q_{F}$ defined by averaging (Clément, 1975). For any $K \in \mathscr{T}_{h}$ we have

$$
\rho_{1, K}^{-1}\left\|\boldsymbol{v}-\boldsymbol{v}_{h}\right\|_{0, K} \lesssim(2 \mu)^{1 / 2}|\boldsymbol{v}|_{1, \omega_{K}}, \quad \rho_{2, K}^{-1}\left\|q_{F}-q_{F, h}\right\|_{0, K} \lesssim(\kappa)^{1 / 2}\left|q_{F}\right|_{1, \omega_{K}},
$$

where $|\cdot|_{1, \omega_{K}}$ is the $H^{1}\left(\omega_{K}\right)$ seminorm. Moreover, for all $E \in \partial K$ we have

$$
\rho_{1, E}^{-1 / 2}\left\|\boldsymbol{v}-\boldsymbol{v}_{h}\right\|_{0, E} \lesssim(2 \mu)^{1 / 2}|\boldsymbol{v}|_{1, \omega_{K}}, \quad \rho_{2, E}^{-1 / 2}\left\|q_{F}-q_{F, h}\right\|_{0, E} \lesssim(\kappa)^{1 / 2}\left|q_{F}\right|_{1, \omega_{K}},
$$

where $\omega_{K}$ is the set of rectangles sharing at least one vertex with $K$.

THEOREM A.1 (Reliability estimate) Suppose that $\left(\boldsymbol{u}, p_{T}, p_{F}\right)$ is the weak solution satisfying (2.1) and that $\left(\boldsymbol{u}_{h}, p_{T, h}, p_{F, h}\right) \in \boldsymbol{X}_{\boldsymbol{u}}^{h} \times M^{h} \times X_{p}^{h}$ is a conforming mixed approximation. Defining $\eta$ and $\Theta$ to be the error estimator and the data oscillation term, we have an upper bound on the approximation error,

$$
\left\|\left(\boldsymbol{u}-\boldsymbol{u}_{h}, p_{T}-p_{T, h}, p_{F}-p_{F, h}\right) \mid\right\| \lesssim \eta+\Theta .
$$

Proof. Since $\left(\boldsymbol{u}-\boldsymbol{u}_{h}, p_{T}-p_{T, h}, p_{F}-p_{F, h}\right) \in \boldsymbol{H}_{\Gamma_{\boldsymbol{u}, 0}}^{1} \times M \times H_{\Gamma_{p, 0}}^{1}$ then from Lemma 2.1, it holds that

$$
\left\|\left(\boldsymbol{u}-\boldsymbol{u}_{h}, p_{T}-p_{T, h}, p_{F}-p_{F, h}\right)\right\|^{2} \lesssim \mathscr{B}\left(\boldsymbol{u}-\boldsymbol{u}_{h}, p_{T}-p_{T, h}, p_{F}-p_{F, h} ; \boldsymbol{v}, q_{T}, q_{F}\right)
$$

with $\left\|\left|\left(\boldsymbol{v}, q_{T}, q_{F}\right)\|\mid \leqslant\|\left(\boldsymbol{u}-\boldsymbol{u}_{h}, p_{T}-p_{T, h}, p_{F}-p_{F, h}\right)\|\|\right.\right.$. Using (2.3) and (2.14) then gives

$$
\begin{aligned}
\mathscr{B}(\boldsymbol{u}- & \left.\boldsymbol{u}_{h}, p_{T}-p_{T, h}, p_{F}-p_{F, h} ; \boldsymbol{v}, q_{T}, q_{F}\right) \\
= & \mathscr{B}\left(\boldsymbol{u}-\boldsymbol{u}_{h}, p_{T}-p_{T, h}, p_{F}-p_{F, h} ; \boldsymbol{v}-\boldsymbol{v}_{h}, q_{T}, q_{F}-q_{F, h}\right) \\
= & \left(\boldsymbol{f}, \boldsymbol{v}-\boldsymbol{v}_{h}\right)-2 \mu\left(\boldsymbol{\varepsilon}\left(\boldsymbol{u}_{h}\right), \boldsymbol{\varepsilon}\left(\boldsymbol{v}-\boldsymbol{v}_{h}\right)\right)+\left(p_{T, h}, \nabla \cdot\left(\boldsymbol{v}-\boldsymbol{v}_{h}\right)\right) \\
& -\left(q_{T}, \nabla \cdot \boldsymbol{u}\right)+\left(q_{T}, \nabla \cdot \boldsymbol{u}_{h}\right)-\frac{1}{\lambda}\left(q_{T}, p_{T}\right)+\frac{1}{\lambda}\left(q_{T}, p_{T, h}\right) \\
& +\frac{\alpha}{\lambda}\left(p_{F}, q_{T}\right)-\frac{\alpha}{\lambda}\left(p_{F, h}, q_{T}\right)+\left(g, q_{F}-q_{F}\right) \\
& +\frac{\alpha^{2}}{\lambda}\left(p_{F, h}, q_{F}-q_{F, h}\right)+\left(\kappa \nabla\left(p_{F, h}\right), \nabla\left(q_{F}-q_{F, h}\right)\right) \\
= & \left(\boldsymbol{f}-\boldsymbol{f}_{h}, \boldsymbol{v}-\boldsymbol{v}_{h}\right)+\left(g-g_{h}, q_{F}-q_{F, h}\right) \\
& +\sum_{K \in \mathscr{T}_{h}}\left\{\left(\nabla \cdot\left(2 \mu \boldsymbol{\varepsilon}\left(\boldsymbol{u}_{h}\right)\right)-\nabla p_{T, h}+\boldsymbol{f}_{h},\left(\boldsymbol{v}-\boldsymbol{v}_{h}\right)\right)_{0, K}\right. \\
& +\sum_{E \in \partial K}\left(\left\langle\boldsymbol{R}_{E}, \boldsymbol{v}-\boldsymbol{v}_{h}\right\rangle_{E}+\left\langle R_{E}, p_{F}-p_{F, h}\right\rangle_{E}\right) \\
& +\left(q_{T}, \nabla \cdot \boldsymbol{u}_{h}+\frac{1}{\lambda} p_{T, h}-\frac{\alpha}{\lambda} p_{F, h}\right)_{0, K} \\
& \left.+\left(g_{h}-\frac{\alpha}{\lambda}\left(p_{T, h}-2 \alpha p_{F, h}\right)-\nabla \cdot\left(\kappa \nabla p_{F, h}\right), q_{F}-q_{F, h}\right)_{0, K}\right\}
\end{aligned}
$$


where $\left\langle\boldsymbol{R}_{E}, \boldsymbol{v}-\boldsymbol{v}_{h}\right\rangle_{E}=\int_{E} \boldsymbol{R}_{E} \cdot\left(\boldsymbol{v}-\boldsymbol{v}_{h}\right)$ and $\left\langle R_{E}, p_{F}-p_{F, h}\right\rangle_{E}=\int_{E} R_{E} \cdot\left(p_{F}-p_{F, h}\right)$. Next, using the Cauchy-Schwarz inequality in (A.2) leads to

$$
\begin{aligned}
\mathscr{B}(\boldsymbol{u}- & \left.\boldsymbol{u}_{h}, p_{T}-p_{T, h}, p_{F}-p_{F, h} ; \boldsymbol{v}, q_{T}, q_{T, h}\right) \\
\leqslant & C\left(\sum_{K \in \mathscr{T}_{h}} \rho_{1, K}^{2}\left\|\boldsymbol{f}-\boldsymbol{f}_{h}\right\|_{0, K}^{2}\right)^{1 / 2}\left(\sum_{K \in \mathscr{T}_{h}} \rho_{1, K}^{-2}\left\|\boldsymbol{v}-\boldsymbol{v}_{h}\right\|_{0, K}^{2}\right)^{1 / 2} \\
& +\left(\sum_{K \in \mathscr{T}_{h}} \rho_{2, K}^{2}\left\|g-g_{h}\right\|_{0, K}^{2}\right)^{1 / 2}\left(\sum_{K \in \mathscr{T}_{h}} \rho_{2, K}^{-2}\left\|q_{F}-q_{F, h}\right\|_{0, K}^{2}\right)^{1 / 2} \\
& +\left(\sum_{K \in \mathscr{T}_{h}} \rho_{1, K}^{2}\left\|\boldsymbol{R}_{K}\right\|_{0, K}^{2}\right)^{1 / 2}\left(\sum_{K \in \mathscr{T}_{h}} \rho_{1, K}^{-2}\left\|\boldsymbol{v}-\boldsymbol{v}_{h}\right\|_{0, K}^{2}\right)^{1 / 2} \\
& +\left(\sum_{K \in \mathscr{T}_{h} \in \sum_{E}} \rho_{1, E}\left\|\boldsymbol{R}_{E}\right\|_{0, E}^{2}\right)^{1 / 2}\left(\sum_{K \in \mathscr{T}_{h} \in \sum_{K}} \rho_{1, E}^{-1}\left\|\boldsymbol{v}-\boldsymbol{v}_{h}\right\|_{0, E}^{2}\right)^{1 / 2} \\
& +\left(\sum_{K \in \mathscr{T}_{h}} \sum_{E \in \partial K} \rho_{2, E}\left\|R_{E}\right\|_{0, E}^{2}\right)^{1 / 2}\left(\sum_{\left.K \in \mathscr{T}_{h} \in \sum_{E} \rho_{2, E}\left\|q_{F}-q_{F, h}\right\|_{0, E}^{2}\right)^{1 / 2}}\right. \\
& +\left(\sum_{K \in \mathscr{T}_{h}} \rho_{d}\left\|\mathscr{R}_{K}\right\|_{0, K}^{2}\right)^{1 / 2}\left(\sum_{K \in \mathscr{T}_{h}} \rho_{d}^{-1}\left\|q_{T}\right\|_{0, K}^{2}\right)^{1 / 2} \\
& \left.+\left(\sum_{K \in \mathscr{T}_{h}} \rho_{2, K}^{2}\left\|R_{K}\right\|_{0, K}^{2}\right)^{1 / 2}\left(\sum_{K \in \mathscr{T}_{h}} \rho_{2, K}^{-2}\left\|q_{F}-q_{F, h}\right\|_{0, K}^{2}\right)^{1 / 2}\right\} .
\end{aligned}
$$

Using Lemma A.1, then leads to the desired upper bound

$$
\begin{aligned}
& \left\|\mid\left(\boldsymbol{u}-\boldsymbol{u}_{h}, p_{T}-p_{T, h}, p_{F}-p_{F, h}\right)\right\|^{2} \\
& \lesssim \mathscr{B}\left(\boldsymbol{u}-\boldsymbol{u}_{h}, p_{T}-p_{T, h}, p_{F}-p_{F, h} ; \boldsymbol{v}, q_{T}, q_{F}\right) \\
& \lesssim\left\{\left(\sum_{K \in \mathscr{T}_{h}} \Theta_{K}^{2}\right)^{1 / 2}\left(\sum_{K \in \mathscr{T}_{h}} \rho_{1, K}^{-2}\left\|\boldsymbol{v}-\boldsymbol{v}_{h}\right\|_{0, K}^{2}+\rho_{2, K}^{-2}\left\|q_{F}-q_{F, h}\right\|_{0, K}^{2}\right)^{1 / 2}\right. \\
& \quad+\left(\sum_{K \in \mathscr{T}_{h}} \eta_{R_{K}}^{2}\right)^{1 / 2}\left(\sum_{K \in \mathscr{T}_{h}} \rho_{1, K}^{-2}\left\|\boldsymbol{v}-\boldsymbol{v}_{h}\right\|_{0, K}^{2}+\rho_{2, K}^{-2}\left\|q_{F}-q_{F, h}\right\|_{0, K}^{2}\right)^{1 / 2} \\
& \quad+\left(\sum_{K \in \mathscr{T}_{h}} \eta_{E_{K}}^{2}\right)^{1 / 2}\left(\sum_{K \in \mathscr{T}_{h} \in \partial K} \sum_{1, E} \rho^{-1}\left\|\boldsymbol{v}-\boldsymbol{v}_{h}\right\|_{0, E}^{2}+\rho_{2, E}^{-1}\left\|q_{F}-q_{F, h}\right\|_{0, E}^{2}\right)^{1 / 2} \\
& \left.\quad+\left(\sum_{K \in \mathscr{T}_{h}} \eta_{J_{K}}^{2}\right)^{1 / 2}\left(\sum_{K \in \mathscr{T}} \rho_{d}^{-1}\left\|q_{T}\right\|_{0, K}^{2}\right)^{1 / 2}\right\} \\
& \lesssim\left(\sum_{K \in \mathscr{T} h}\left\{2 \mu|\boldsymbol{v}|_{1, K}^{2}+\rho_{d}^{-1}\left\|q_{T}\right\|_{0, K}^{2}+\left\|\kappa \nabla q_{F}\right\|_{0, K}^{2}\right\}\right)^{\frac{1}{2}}\left(\sum_{K \in \mathscr{T} h}\left(\eta_{K}^{2}+\Theta_{K}^{2}\right)\right)^{\frac{1}{2}}
\end{aligned}
$$

Thus,

$$
\left.||\left|\left(\boldsymbol{u}-\boldsymbol{u}_{h}, p_{T}-p_{T, h}, p_{F}-p_{F, h}\right)\right|\right|^{2} \lesssim\left\|||\left(\boldsymbol{v}, q_{T}, q_{F}\right) \mid\right\|\left(\sum_{K \in \mathscr{T} h}\left(\eta_{K}^{2}+\Theta_{K}^{2}\right)\right)^{\frac{1}{2}}
$$


as required.

Next, let $K$ be an element of $\mathscr{T}_{h}$ and suppose that $\chi_{K}$ is a (quartic) interior bubble function (positive in the interior of $K$, zero on $\partial K$ ). Then the following estimates hold (Verfürth, 2013).

$$
\begin{aligned}
\left\|\chi_{K} v\right\|_{0, K} & \lesssim\|v\|_{0, K} \lesssim\left\|\chi_{K}^{1 / 2} v\right\|_{0, K}, \\
\left\|\nabla\left(\chi_{K} v\right)\right\|_{0, K} & \lesssim h_{K}^{-1}\|v\|_{0, K},
\end{aligned}
$$

where $v$ denotes a scalar-valued polynomial function defined on $K$. The efficiency of the residual error estimator is a consequence of the following sequence of lemmas.

LEMMA A.2 Let $K$ be an element of $\mathscr{T}_{h}$. The local momentum residual satisfies

$$
\rho_{1, K}^{2}\left\|\boldsymbol{R}_{K}\right\|_{0, K}^{2} \lesssim 2 \mu\left|\boldsymbol{u}-\boldsymbol{u}_{h}\right|_{1, K}^{2}+(2 \mu)^{-1}\left\|p_{T}-p_{T, h}\right\|_{0, K}^{2}+\Theta_{K}^{2} .
$$

Proof. See Khan et al. (2017).

Lemma A.3 Let $K$ be an element of $\mathscr{T}_{h}$. The local mass conservation residual satisfies

$$
\rho_{2, K}^{2}\left\|R_{K}\right\|_{0, K}^{2} \lesssim\left\|\kappa^{\frac{1}{2}} \nabla\left(p_{F}-p_{F, h}\right)\right\|_{0, K}^{2}+\frac{1}{\lambda}\left\|p_{T}-p_{T, h}\right\|_{0, K}^{2}+\frac{\alpha}{\lambda}\left\|p_{F}-p_{F, h}\right\|_{0, K}^{2}+\Theta_{K}^{2}
$$

Proof. For each element $K$ in $\mathscr{T}_{h}$, we have $R_{K}=\left.\left(g_{h}-\frac{\alpha}{\lambda}\left(p_{T, h}-2 \alpha p_{F, h}\right)-\nabla \cdot\left(\kappa \nabla p_{F, h}\right)\right)\right|_{K}$. Next, introducing $\left.w\right|_{K}=\rho_{2, K}^{2} R_{K} \chi_{K}$ and using (A.5) we have

$$
\rho_{2, K}^{2}\left\|R_{K}\right\|_{0, K}^{2} \lesssim\left(R_{K}, \rho_{2, K}^{2} \chi_{K} R_{K}\right)_{K}=\left(g_{h}-\frac{\alpha}{\lambda}\left(p_{T, h}-2 \alpha p_{F, h}\right)-\nabla \cdot\left(\kappa \nabla p_{F, h}\right), w\right)_{K} .
$$

Using integration by parts with $\left.w\right|_{\partial K}=0$ and (2.1c) implies

$$
\rho_{2, K}^{2}\left\|R_{K}\right\|_{0, K}^{2}=\left(\kappa \nabla\left(p_{F, h}-p_{F}\right), \nabla w\right)_{K}+\frac{\alpha}{\lambda}\left(p_{T}-p_{T, h}, w\right)_{K}-\frac{2 \alpha^{2}}{\lambda}\left(p_{F}-p_{F, h}, w\right)_{K}+\left(g_{h}-g, w\right)_{K} .
$$

Applying the Cauchy-Schwarz inequality leads to the bound

$$
\begin{gathered}
\rho_{2, K}^{2}\left\|R_{K}\right\|_{0, K}^{2} \lesssim\left(\left\|\kappa^{1 / 2} \nabla\left(p_{F}-p_{F, h}\right)\right\|_{0, K}^{2}+\frac{1}{\lambda}\left\|p_{T}-p_{T, h}\right\|_{0, K}^{2}+\frac{\alpha}{\lambda}\left\|p_{F}-p_{F, h}\right\|_{0, K}^{2}\right. \\
\left.+\rho_{2, K}^{2}\left\|g-g_{h}\right\|_{0, K}^{2}\right)^{\frac{1}{2}}\left(\left\|\kappa^{1 / 2} \nabla w\right\|_{0, K}^{2}+\rho_{2, K}^{-2}\|w\|_{0, K}^{2}\right)^{\frac{1}{2}} .
\end{gathered}
$$

Using (A.5) and (A.6) in (A.7) then gives the bound

$\rho_{2, K}^{2}\left\|R_{K}\right\|_{0, K}^{2} \lesssim\left(\left\|\kappa^{\frac{1}{2}} \nabla\left(p_{F}-p_{F, h}\right)\right\|_{0, K}^{2}+\frac{1}{\lambda}\left\|p_{T}-p_{T, h}\right\|_{0, K}^{2}+\frac{\alpha}{\lambda}\left\|p_{F}-p_{F, h}\right\|_{0, K}^{2}+\Theta_{K}^{2}\right)^{\frac{1}{2}}\left(\rho_{2, K}^{2}\left\|R_{K}\right\|_{0, K}^{2}\right)^{\frac{1}{2}}$

as required.

Lemma A.4 Let $K$ be an element of $\mathscr{T}_{h}$. The local pressure balance residual satisfies

$$
\eta_{J_{K}}^{2} \lesssim\left(2 \mu\left|\boldsymbol{u}-\boldsymbol{u}_{h}\right|_{1, K}^{2}+\left(\frac{1}{2 \mu}+\frac{1}{\lambda}\right)\left\|p_{T}-p_{T, h}\right\|_{0, K}^{2}+\frac{\alpha}{\lambda}\left\|p_{F}-p_{F, h}\right\|_{0, K}^{2}\right) .
$$


Proof. Noting that $\left.\left(\nabla \cdot \boldsymbol{u}+\frac{1}{\lambda} p_{T}-\frac{\alpha}{\lambda} p_{F}\right)\right|_{K}=0$ for the exact solution $\left(\boldsymbol{u}, p_{T}, p_{F}\right)$, we have

$$
\begin{aligned}
& \rho_{d}\left\|\nabla \cdot \boldsymbol{u}_{h}+\frac{1}{\lambda} p_{T, h}-\frac{\alpha}{\lambda}\right\|_{0, K}^{2}=\rho_{d}\left\|\nabla \cdot\left(\boldsymbol{u}-\boldsymbol{u}_{h}\right)+\frac{1}{\lambda}\left(p_{T}-p_{T, h}\right)-\frac{\alpha}{\lambda}\left(p_{F}-p_{F, h}\right)\right\|_{0, K}^{2} \\
& \lesssim \rho_{d}\left\|\nabla \cdot\left(\boldsymbol{u}-\boldsymbol{u}_{h}\right)\right\|_{0, K}^{2}+\frac{\rho_{d}}{\lambda^{2}}\left\|\left(p_{T}-p_{T, h}\right)\right\|_{0, K}^{2} \\
& \quad+\frac{\rho_{d} \alpha^{2}}{\lambda^{2}}\left\|\left(p_{F}-p_{F, h}\right)\right\|_{0, K}^{2}, \\
& \lesssim 2 \mu\left|\boldsymbol{u}-\boldsymbol{u}_{h}\right|_{1, K}^{2}+\frac{1}{\lambda}\left\|p_{T}-p_{T, h}\right\|_{0, K}^{2}+\frac{\alpha^{2}}{\lambda}\left\|p_{F}-p_{F, h}\right\|_{0, K}^{2},
\end{aligned}
$$

where the last line follows from the definition of $\rho_{d}$ in (3.1).

Next, let $E$ denote an interior edge which is shared by two elements $K$ and $K^{\prime}$ and suppose that $\chi_{E}$ is a polynomial bubble function on $E$ (positive in the interior of the patch $\omega_{E}$ formed by the union of $K$ and $K^{\prime}$ and zero on the boundary of the patch). The following estimates are also due to Verfürth (2013),

$$
\begin{aligned}
\|q\|_{0, E} & \lesssim\left\|\chi_{E}^{1 / 2} q\right\|_{0, E} \\
\left\|\chi_{E} q\right\|_{0, K} & \lesssim h_{E}^{1 / 2}\|q\|_{0, E} \quad \forall K \in \omega_{E} \\
\left\|\nabla\left(\chi_{E} q\right)\right\|_{0, K} & \lesssim h_{E}^{-1 / 2}\|q\|_{0, E} \quad \forall K \in \omega_{E} .
\end{aligned}
$$

Here, $q$ is a scalar-valued polynomial function defined on $E$ and $q_{\chi}=\chi_{E} q$ can be extended by zero outside of the patch.

LEMmA A.5 Let $K$ be an element of $\mathscr{T}_{h}$. The stress jump residual satisfies

$$
\sum_{E \in \partial K} \rho_{1, E}\left\|\boldsymbol{R}_{E}\right\|_{0, E}^{2} \lesssim \sum_{E \in \partial K}\left(2 \mu\left|\boldsymbol{u}-\boldsymbol{u}_{h}\right|_{1, \omega_{E}}^{2}+(2 \mu)^{-1}\left\|p_{T}-p_{T, h}\right\|_{0, \omega_{E}}^{2}+\Theta_{\omega_{E}}^{2}\right),
$$

where $\Theta_{\omega_{E}}^{2}=\sum_{K \in \omega_{E}} \Theta_{K}^{2}$ is the localised data oscillation term.

Proof. See Khan et al. (2017).

LEMmA A.6 Let $K$ be an element of $\mathscr{T}_{h}$. The fluid flux jump residual satisfies

$$
\sum_{E \in \partial K} \rho_{2, E}\left\|R_{E}\right\|_{0, E}^{2} \lesssim \sum_{E \in \partial K}\left(2 \mu\left|\boldsymbol{u}-\boldsymbol{u}_{h}\right|_{1, \omega_{E}}^{2}+(2 \mu)^{-1}\left\|p-p_{h}\right\|_{0, \omega_{E}}^{2}+\Theta_{\omega_{E}}^{2}\right),
$$

where $\Theta_{\omega_{E}}^{2}=\sum_{K \in \omega_{E}} \Theta_{K}^{2}$ is the localised data oscillation term.

Proof. Suppose $E$ is an interior edge and let $\chi_{E}$ be a polynomial bubble function associated with $E$ as defined above. We define the localised jump term so that $\Lambda=\rho_{E} \llbracket\left(\kappa \nabla p_{F, h}\right) \cdot \boldsymbol{n} \rrbracket \chi_{E}$. Using (A.9) and (3.7) gives the estimate

$$
\rho_{2, E}\left\|R_{E}\right\|_{0, E}^{2} \lesssim\left(\llbracket\left(\kappa \nabla p_{F, h}\right) \cdot \boldsymbol{n} \rrbracket, \Lambda\right)_{E}
$$

Then, using (2.1c) for every $q_{F} \in H_{\Gamma_{p, 0}}^{1}$ we have

$$
\int_{\Omega} \kappa(\boldsymbol{x}) \nabla\left(p_{F}-p_{F, h}\right) \cdot \nabla q_{F}=\frac{\alpha}{\lambda} \int_{\Omega} p_{T} q_{F}-\frac{2 \alpha}{\lambda} \int_{\Omega} p_{F} q_{F}-\int_{\Omega} g q_{F}-\int_{\Omega} \nabla p_{F, h} \cdot \nabla q_{F} .
$$


Integration by parts elementwise gives

$$
\int_{\Omega} g q_{F}+\int_{\Omega} \nabla p_{F, h} \cdot \nabla q_{F}=\sum_{K \in \mathscr{T}_{h}} \int_{K}\left(g-\nabla \cdot\left(\kappa \nabla p_{F, h}\right)\right) q_{F}+\sum_{E \in \mathscr{E}\left(\mathscr{T}_{h}\right)} \int_{E} \llbracket\left(\kappa \nabla p_{F, h}\right) \cdot \boldsymbol{n} \rrbracket q_{F} .
$$

Choosing the test function $q_{F}=\Lambda$ in (A.13) and (A.14), and then combining (A.12), (A.13) and (A.14), we have

$$
\begin{aligned}
\rho_{2, E}\left\|R_{E}\right\|_{0, E}^{2} \lesssim & \sum_{K \in \omega_{E}} \int_{K}\left\{g_{h}-\alpha \lambda^{-1}\left(p_{T, h}-2 \alpha p_{F, h}\right)-\nabla \cdot\left(\kappa \nabla\left(p_{F, h}\right)\right)\right\} \Lambda \\
& +\sum_{K \in \omega_{E}} \int_{K}\left\{\left(g-g_{h}\right)-\alpha \lambda^{-1}\left(p_{T}-p_{T, h}\right)+2 \alpha^{2} \lambda^{-2}\left(p_{F}-p_{F, h}\right)\right\} \Lambda \\
& +\sum_{K \in \omega_{E}} \int_{K}\left\{-\kappa\left(\nabla p_{F}-\nabla p_{F, h}\right)\right\} \cdot \nabla \Lambda \\
\lesssim & T_{1}+T_{2}+T_{3} .
\end{aligned}
$$

These three terms will be bounded separately.

First, using the definition of $R_{K}$ and applying the Cauchy-Schwarz inequality with Lemma A.3 gives

$$
\begin{aligned}
T_{1} \lesssim( & \left.\frac{1}{\lambda}\left\|p_{T}-p_{T, h}\right\|_{0, \omega_{E}}^{2}+\frac{\alpha^{2}}{\lambda}\left\|p_{F}-p_{F, h}\right\|_{0, \omega_{E}}^{2}+\left\|\kappa\left(\nabla p_{F}-\nabla p_{F, h}\right)\right\|_{0, \omega_{E}}^{2}+\Theta_{\omega_{E}}^{2}\right)^{1 / 2} \\
& \left(\sum_{K \in \omega_{E}} \rho_{K}^{-2}\|\Lambda\|_{0, K}^{2}\right)^{1 / 2} .
\end{aligned}
$$

Next, given the shape regularity of the grid, using the definition of $\Lambda$ and (A.10) gives

$$
\rho_{2, K}^{-2}\|\Lambda\|_{0, K}^{2} \lesssim \rho_{2, E}^{-1} h_{E}^{-1}\|\Lambda\|_{0, K}^{2} \lesssim \rho_{2, E}^{-1}\left\|\rho_{2, E} R_{E}\right\|_{0, E}^{2} .
$$

Hence, the following estimate holds

$$
\begin{aligned}
T_{1} \lesssim( & \left.\frac{1}{\lambda}\left\|p_{T}-p_{T, h}\right\|_{0, \omega_{E}}^{2}+\frac{\alpha^{2}}{\lambda}\left\|p_{F}-p_{F, h}\right\|_{0, \omega_{E}}^{2}+\left\|\kappa^{\frac{1}{2}}\left(\nabla p_{F}-\nabla p_{F, h}\right)\right\|_{0, \omega_{E}}^{2}+\Theta_{\omega_{E}}^{2}\right)^{\frac{1}{2}} \\
& \left(\sum_{K \in \omega_{E}} \rho_{E}\left\|R_{E}\right\|_{0, E}^{2}\right)^{1 / 2} \cdot
\end{aligned}
$$

Second, combining the Cauchy-Schwarz inequality with the above construction gives

$$
\begin{aligned}
T_{2} & \lesssim\left(\sum_{K \in \omega_{E}} \rho_{2, K}^{2}\left\|g-g_{h}\right\|_{0, K}^{2}+\frac{1}{\lambda}\left\|p_{T}-p_{T, h}\right\|_{0, \omega_{E}}^{2}+\frac{\alpha^{2}}{\lambda}\left\|p_{F}-p_{F, h}\right\|_{0, \omega_{E}}^{2}\right)^{1 / 2}\left(\sum_{K \in \omega_{E}} \rho_{K}^{-2}\|\Lambda\|_{0, K}^{2}\right)^{1 / 2} \\
& \lesssim\left(\Theta_{\omega_{E}}^{2}+\frac{1}{\lambda}\left\|p_{T}-p_{T, h}\right\|_{0, \omega_{E}}^{2}+\frac{\alpha^{2}}{\lambda}\left\|p_{F}-p_{F, h}\right\|_{0, \omega_{E}}^{2}\right)^{\frac{1}{2}}\left(\sum_{K \in \omega_{E}} \rho_{E}\left\|R_{E}\right\|_{0, E}^{2}\right)^{1 / 2}
\end{aligned}
$$

The third term can be bounded in a similar way,

$$
T_{3} \lesssim\left\|\kappa^{\frac{1}{2}}\left(\nabla p_{F}-\nabla p_{F, h}\right)\right\|_{0, \omega_{E}}\left(\sum_{K \in \omega_{E}}\left\|\kappa^{1 / 2} \nabla \Lambda\right\|_{0, K}^{2}\right)^{1 / 2}
$$


where this time the second term is bounded using (A.11),

$$
\left\|\kappa^{\frac{1}{2}} \nabla \Lambda\right\|_{0, K}^{2} \lesssim \rho_{2, E}^{-1} h_{E}\|\nabla \Lambda\|_{0, K}^{2} \lesssim \rho_{2, E}^{-1}\left\|\rho_{2, E} R_{E}\right\|_{0, E}^{2} .
$$

Combining the upper bounds for $T_{1}, T_{2}$ and $T_{3}$, for any interior edge $E$, we have

$$
\rho_{2, E}\left\|R_{E}\right\|_{0, E}^{2} \lesssim\left(\frac{1}{\lambda}\left\|p_{T}-p_{T, h}\right\|_{0, \omega_{E}}^{2}+\frac{\alpha^{2}}{\lambda}\left\|p_{F}-p_{F, h}\right\|_{0, \omega_{E}}^{2}+\left\|\kappa^{\frac{1}{2}}\left(\nabla p_{F}-\nabla p_{F, h}\right)\right\|_{0, \omega_{E}}^{2}+\Theta_{\omega_{E}}^{2}\right) .
$$

If $E \in \Gamma_{N}$, then the same result holds with $\omega_{E}=K$ and we recall from (3.7) that $R_{E}=0$ for edges $E$ on $\Gamma_{D}$. Hence, summing over all the edges of element $K$ gives the required result.

Combining Lemmas A.2-A.6 leads to the efficiency bound.

THEOREM A.2 Suppose that $\left(\boldsymbol{u}, p_{T}, p_{F}\right)$ is the weak solution satisfying (2.1) and that $\left(\boldsymbol{u}_{h}, p_{T, h}, p_{F, h}\right) \in$ $\boldsymbol{X}_{\boldsymbol{u}}^{h} \times M^{h} \times X_{p}^{h}$ is a conforming mixed approximation. Defining $\eta$ and $\Theta$ to be the error estimator and the data oscillation term we have a lower bound on the approximation error,

$$
\eta \lesssim\left\|||\left(\boldsymbol{u}-\boldsymbol{u}_{h}, p_{T}-p_{T, h}, p_{F}-p_{F, h}\right) \mid\right\|+\Theta .
$$

